\title{
Earth-based observations of impact phenomena
}

\author{
By PHILIP D. NICHOLSON
}

Department of Astronomy, Cornell University, Ithaca, NY, 14853, USA

Earth-based observations at near- and mid-infrared wavelengths were obtained for at least 15 of the SL9 impacts, ranging from the spectacular G, K and L events to the barely-detected N and $\mathrm{V}$ impacts. Although there were a few exceptions, most of the IR lightcurves fit a common pattern of one or two relatively faint precursor flashes, followed several minutes later by the main infrared event as the explosively-ejected plume crashed down onto the jovian atmosphere. Correlations with the impact times recorded by the Galileo spacecraft and plumes imaged by the Hubble Space Telescope lead to an interpretation of the twin precursors in terms of (i) the entry of the bolide into the upper atmosphere, and (ii) the re-appearance of the rising fireball above Jupiter's limb. Positive correlations are observed between the peak IR flux observed during the splashback phase and both pre-impact size estimates for the individual SL9 fragments and the scale of the resulting ejecta deposits. None of the fragments observed to have moved off the main train of the comet by May 1994 produced a significant impact signature. Earth-based fireball temperature estimates are on the order of $750 \mathrm{~K}, 30-60 \mathrm{sec}$ after impact. For the larger impacts, the unexpectedly protracted fireball emission at $2.3 \mu \mathrm{m}$ remains unexplained. A wide range of temperatures has been inferred for the splashback phase, where shocks are expected to have heated the re-entering plume material at least briefly to several thousand $\mathrm{K}$, and further modelling is required to reconcile these data.

\section{Introduction}

The impacts of the 20 or so fragments of comet Shoemaker-Levy 9 (henceforth SL9 for simplicity) with Jupiter in July 1994 were observed from a wide range of Earth-based telescopes, including instruments in Europe, South and North America, Hawaii, Australia, Japan, South Africa and even Antarctica. In this review we will concentrate on Earth-based near-infrared and mid-infrared observations of prompt phenomena, defined as those occurring within approximately one hour after the impacts. The extensive sets of post-impact imaging and spectroscopic studies of the impact sites, including compositional analyses of the impact debris, are reviewed in the chapters by West, Lellouch, Moses, and Conrath. Magnetospheric effects are reviewed in the chapter by Ip.

The most extensive sets of ground-based data were obtained in the near-infrared, especially at wavelengths of 2.3 and $3.5 \mu \mathrm{m}$ where methane absorption bands in the jovian spectrum greatly reduce the planetary background and enhance the contrast of the impact features. By a fortunate coincidence, this spectral region was also near the peak of thermal emission from the impact plumes and their remnants. A new generation of infrared cameras and spectrometers provided the bulk of the data, and we may reflect upon our good fortune that the impact of SL9 did not occur a decade earlier.

Although Jupiter rose in the early afternoon and set before midnight at most sites (the notable exception being the South Pole station where almost continuous monitoring was possible), the wide geographical distribution of observers ensured that attempts were made to observe every predicted impact. Of the 24 predicted impacts (Sekanina et al. 1994; Chodas \& Yeomans 1994 [email predictions]), convincing detections of 15 have been reported to date, and another three ( $B, M$ and $U$ ) may have been marginally detected. Six fragments (F, G2, J, P1, P2 and T) appear either to have disintegrated completely 
before encountering Jupiter, or to have disappeared into the planet's atmosphere without a trace.

After a discussion of a typical SL9 lightcurve, and what has become the standard interpretation of its main features, we begin in $\S 2$ by summarizing the observed phenomena and classifying the impacts into five categories. In $\S 3$ we present a detailed description of the successive phases in a generic lightcurve, drawing on individual examples for illustrations. An attempt is made to relate these observations to those made simultaneously by the Galileo spececraft (see chapter by Chapman in this volume) and by the Hubble Space Telescope (see chapter by Hammel). A simple ballistic model of the impact plumes which is consistent with the ensemble of observed lightcurves is used as common framework for interpretation of the observations. This model is based loosely on the numerical models developed in the chapters by Crawford, Zahnle and MacLow. In $\S 4$ we discuss the relatively small number of spectroscopic observations made during the actual impact events, and their interpretation in terms of plume and fallback temperatures. Sections 5 and 6 cover other prompt phenomena associated with the impacts, while in $\S 7$ we summarize our conclusions.

\subsection{A typical example: the $R$ impact}

Most, if not all, of the features seen in the near-infrared SL9 impact lightcurves are illustrated in the data obtained for the medium-sized $\mathrm{R}$ impact and shown in Figure 1. The $2.3 \mu \mathrm{m}$ observations were made at Mauna Kea with the Keck $10 \mathrm{~m}$ telescope and the near-IR camera (Graham et al. 1995), while the 3.2 and $4.5 \mu \mathrm{m}$ observations were made at the $5 \mathrm{~m}$ Hale telescope at Palomar, using simultaneously-mounted near-IR and mid-IR cameras (Nicholson et al. 1995a). Sampling times were 8-10 sec at 2.3 and $4.5 \mu \mathrm{m}$ and $30 \mathrm{sec}$ at $3.2 \mu \mathrm{m}$. The $4.5 \mu \mathrm{m}$ fluxes have been corrected for background light from Jupiter, but the steady pre-impact slope in the $2.3 \mu \mathrm{m}$ data is due to the rotation of the older $\mathrm{G}$ impact site onto the jovian limb. Black bars under the lightcurves indicate the times (corrected for light travel time) during which the Galileo NIMS instrument observed detectable flux from the impact (Carlson et al. 1995b).

Shortly before the initial Galileo flash, a brief flash was observed at all three wavelengths with a duration of $\sim 30 \mathrm{sec}$. About $60 \mathrm{sec}$ after the first signal, a second, brighter flash began abruptly, only to decay over the next minute or so (within $30 \mathrm{sec}$ at $4.5 \mu \mathrm{m}$, but extending over $3 \mathrm{~min}$ at $2.3 \mu \mathrm{m}$ ). These two events have come to be known as the first and second precursors, respectively. The precursor events are shown at an expanded scale in Fig. 1(b). Approximately $6 \mathrm{~min}$ after the initial flash a dramatic brightening commenced, eventually reaching a peak flux about one hundred times greater than that of the second precursor, $10 \mathrm{~min}$ after the impact. This main event was observed simultaneously by earth-based telescopes and by Galileo NIMS. Following the peak of the main event the infrared flux decayed with a time constant of $\sim 3 \mathrm{~min}$. This fading was interrupted by a shoulder, or secondary maximum, occuring $\sim 9 \mathrm{~min}$ after the peak. At $3.2 \mu \mathrm{m}$ there is an indication of a second, weaker shoulder $\sim 18 \mathrm{~min}$ after the main peak.

The generic sequence of events represented by this lightcurve is illustrated schematically in Fig. 1 in the chapter by Zahnle, and in Fig. 2 of Boslough et al. (1995). Figure 2 shows the particular geometry for earth-based observations of the $\mathrm{R}$ impact. As seen from Earth, the impact itself occurred $\sim 5.7^{\circ}$ in longitude behind the planet's dawn limb; the events were observed directly only by Galileo. The first precursor apparently corresponded to thermal emission from the trail left by the passage of the fragment through Jupiter's upper atmosphere, and was too faint to be detected by the less-sensitive Galileo instruments. About $30 \mathrm{sec}$ after Galileo observed the intensely bright terminal phase of the impact, the expanding, incandescent fireball rose above the limb into our 

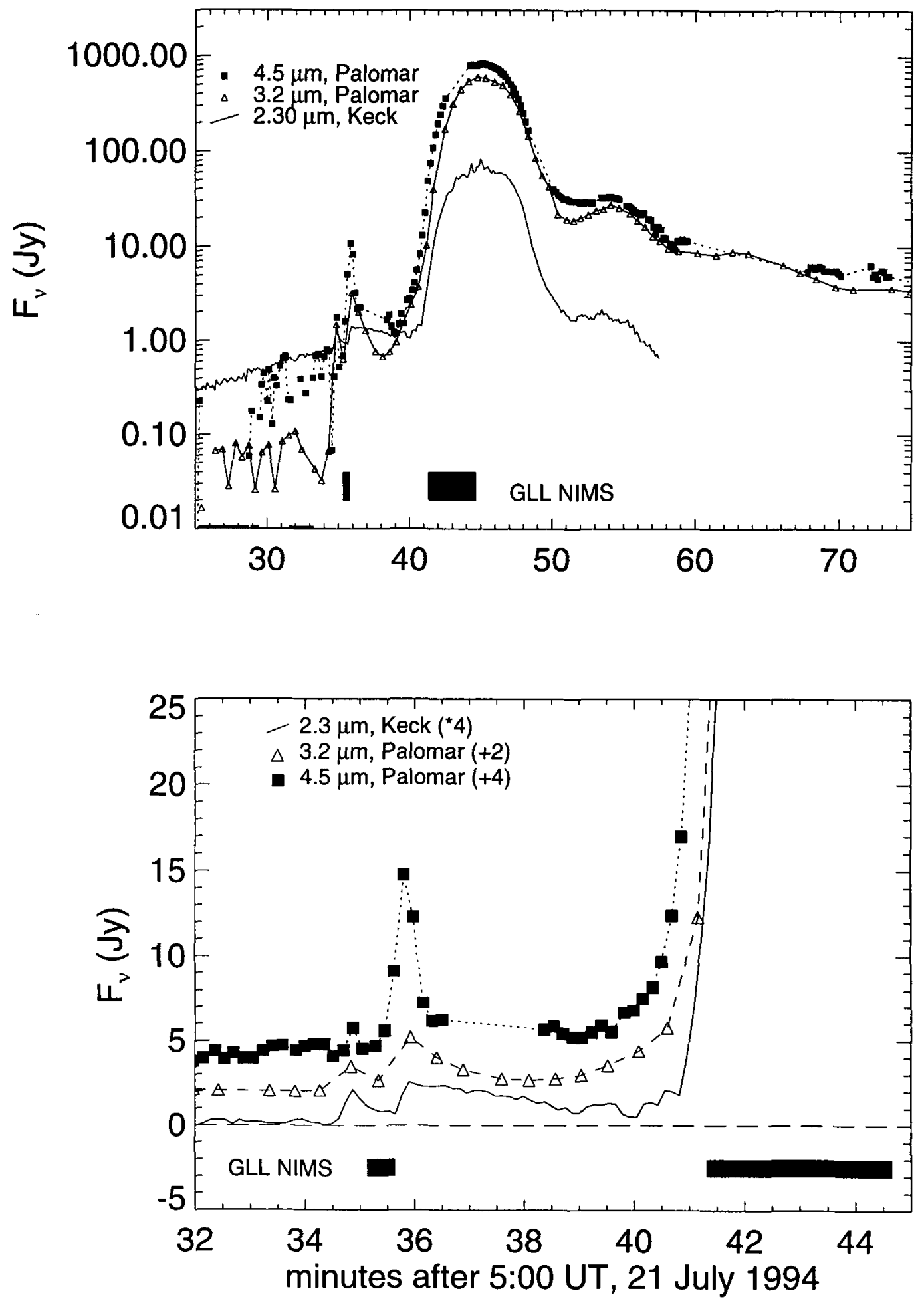

FIgURE 1. (a) Lightcurves for the $\mathrm{R}$ impact, as observed at the Keck $10 \mathrm{~m}$ and Palomar $5 \mathrm{~m}$ telescopes, on a logarithmic scale. Gaps in the $4.5 \mu \mathrm{m}$ data correspond to periods during which spectroscopic measurements were obtained. Solid black bars indicate the periods of emission observed by the Galileo NIMS instrument. (b) Expanded plot of the R precursor events on a linear scale. Note the scale factor applied to the $2.3 \mu \mathrm{m}$ data. 


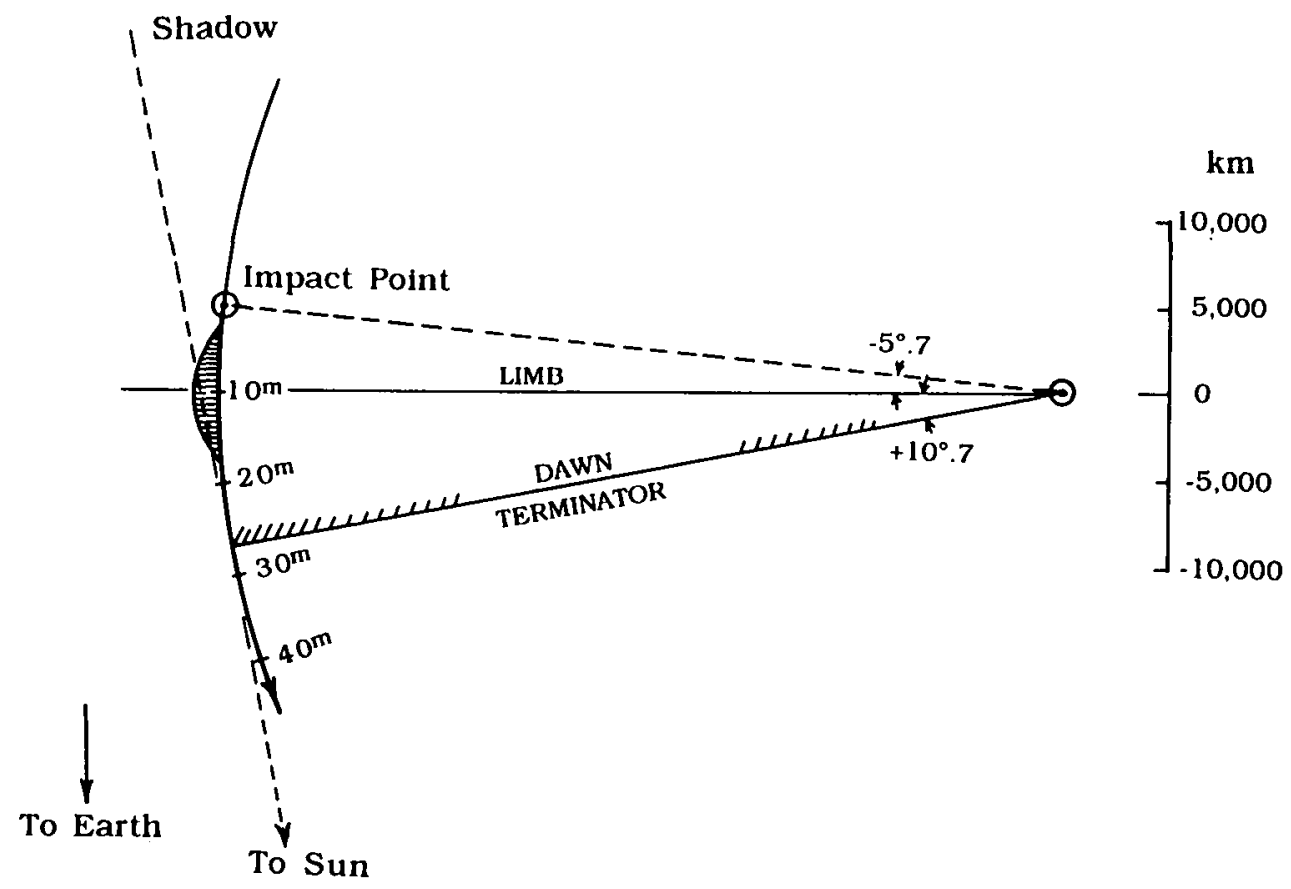

FIgURE 2. Geometry for earth-based observations of the $R$ impact. The diagram shows the view from above Jupiter's north pole, with the Earth towards the bottom of the page and the planet's center towards the right edge. The $\mathrm{R}$ impact site, indicated by the $\odot$ symbol, was behind the limb and out of direct sight. Ten min after the impact, and near the time of peak IR flux, the site reached the limb. The plume was also near its maximum height, as indicated schematically by the hatched region. Another $17 \mathrm{~min}$ elapsed before the impact site reached the dawn terminator and direct sunlight. The geometry for the other impacts was similar, with earlier impacts generally occurring further behind the limb and later impacts even closer (Sekanina et al. 1994).

line of sight, although still in the pre-dawn shadow. This is the second precursor, whose decay represents the rapidly cooling fireball. Now quite cold, but emerging after 1-2 min into sunlight and visibility by the Hubble Space Telescope (henceforth HST), the debris plume reached a maximum altitude of $\sim 3200 \mathrm{~km}$ above Jupiter's cloud tops (Hammel et. al. 1995), before falling back into the planet's atmosphere. (This is based on observations of other impacts; HST did not observe the R impact.) For R, the maximum height was reached just as the impact site itself rotated into view, but this was not the case for earlier impacts, which occurred further behind the limb. The infrared main event corresponds to this extended period of fallback, which lasted from 6 to $\sim 15 \min$ after the impact, and during which the descending debris was shock-heated again to temperatures of at least $500-1000 \mathrm{~K}$, and perhaps much higher.

The origin of the secondary shoulders is less certain, but they may be due to reentering material 'bouncing' off the top of the atmosphere and re-entering a second (or even a third) time (Deming et al. 1995). Not until $\sim 27 \mathrm{~min}$ after the impact did the $\mathrm{R}$ impact site cross the dawn terminator and emerge into direct sunlight. In practice, it is impossible to distinguish in near-IR images of the impacts between the dying thermal stages of the main event and the appearance of the fresh impact site in reflected sunlight on the terminator. 
With minor modifications, this scenario seems to fit the observations of almost all of the impacts, while also being consistent with numerical models of the process of fireball formation and evolution (Boslough et al. 1994; Crawford et al. 1994; Zahnle \& MacLow 1994; Takata et al. 1994). We will use it as a working model in describing some of the detailed features exhibited by other lightcurves in $\S 3$ below.

\section{Lightcurve classification}

\subsection{Lightcurve comparisons}

Comparison of lightcurves for different events is often complicated by differences in telescope aperture, wavelength of observation, sampling time and weather conditions. Two extensive series of homogeneous observations which avoid these pitfalls are shown in Figs. 3 and 4. Figure 3, from McGregor et al. (1995), presents near-IR lightcurves obtained at the $2.3 \mathrm{~m}$ telescope at Siding Spring Observatory, Australia for events C, D, G, $\mathrm{K}, \mathrm{R}$ and $\mathrm{W}$. Impact $\mathrm{N}$ was detected only weakly in 3 frames, while $\mathrm{V}$ was not detected at all. The data were obtained with the CASPIR near-IR camera, at exposure intervals of $\sim 50 \mathrm{sec}$, using a narrow-band $2.34 \mu \mathrm{m}$ filter. These data include examples of large, medium-size and small impacts, and in addition to precursors for events C, D, G, K and W, the lightcurves show prominent shoulders for C, D and G. (The lightcurves for the later impacts were followed only through the main peak.)

Figure 4, from Lagage et al. (1995), presents a series of mid-IR lightcurves for the A, $\mathrm{E}, \mathrm{H}, \mathrm{L}$ and Q1 impacts obtained with the $10 \mu \mathrm{m}$ CAMIRAS camera at the $2.6 \mathrm{~m}$ Nordic Optical Telescope at La Palma, in the Canary Islands. Impacts F, P2, Q2, T and U were not detected. The filter passband was $10.5-13 \mu \mathrm{m}$, and the original $1.1 \mathrm{sec}$ samples have been binned at intervals of $\sim 60 \mathrm{sec}$. The background thermal emission from Jupiter has been subtracted from the images. The lightcurve for the extremely bright $\mathrm{L}$ impact also shows a brief precursor $\sim 60 \mathrm{sec}$ after the Galileo flash, with a peak flux of $300 \mathrm{Jy}$. A similar precursor was seen at $10 \mu \mathrm{m}$ for the $\mathrm{H}$ impact by Livengood et al. (1995), using the TIMMI camera at the ESO $3.6 \mathrm{~m}$ telescope at La Silla, Chile. Although not prominent in this presentation, each of the lightcurves shows a definite shoulder $\sim 20 \mathrm{~min}$ after the impact. The shoulders are most prominent for $\mathrm{H}$ and $\mathrm{L}$.

\subsection{Summary of observations}

Table 1, compiled by P. Chodas from published reports and a survey of participants at IAU Colloquium 156, and with some additions by the author, summarizes the observed phenomena associated with each impact. The adopted impact times are based on Galileo observations of the initial flash where available, on Earth-based precursor times, orwhere necessary - on predictions or extrapolation from the onset of the of the main event. For further details the chapter by Chodas \& Yeomans should be consulted. PC1 and PC2 refer to detections of the first and second precursors, respectively, and ME to observations of the infrared main event. The columns labelled 'spot' and 'ejecta' refer to the presence of these features in HST images (Hammel et al. 1995). The last two columns give the morphological class assigned by Hammel et al., which reflects a combination of characteristics such as central spot size, scale of ejecta blanket, and prominence of waves (if any), and the Galileo instruments which observed the event, where applicable.

In Fig. 5 we plot the main event peak flux at $2.3 \mu \mathrm{m}$, a quantity which is available for most of the observed impacts from published lightcurves, vs. the HST class. On the basis of this diagram, and on the observability of the post-impact features in ground-based images of Jupiter, the impacts can be fairly cleanly (if somewhat arbitrarily) divided into five categories. 

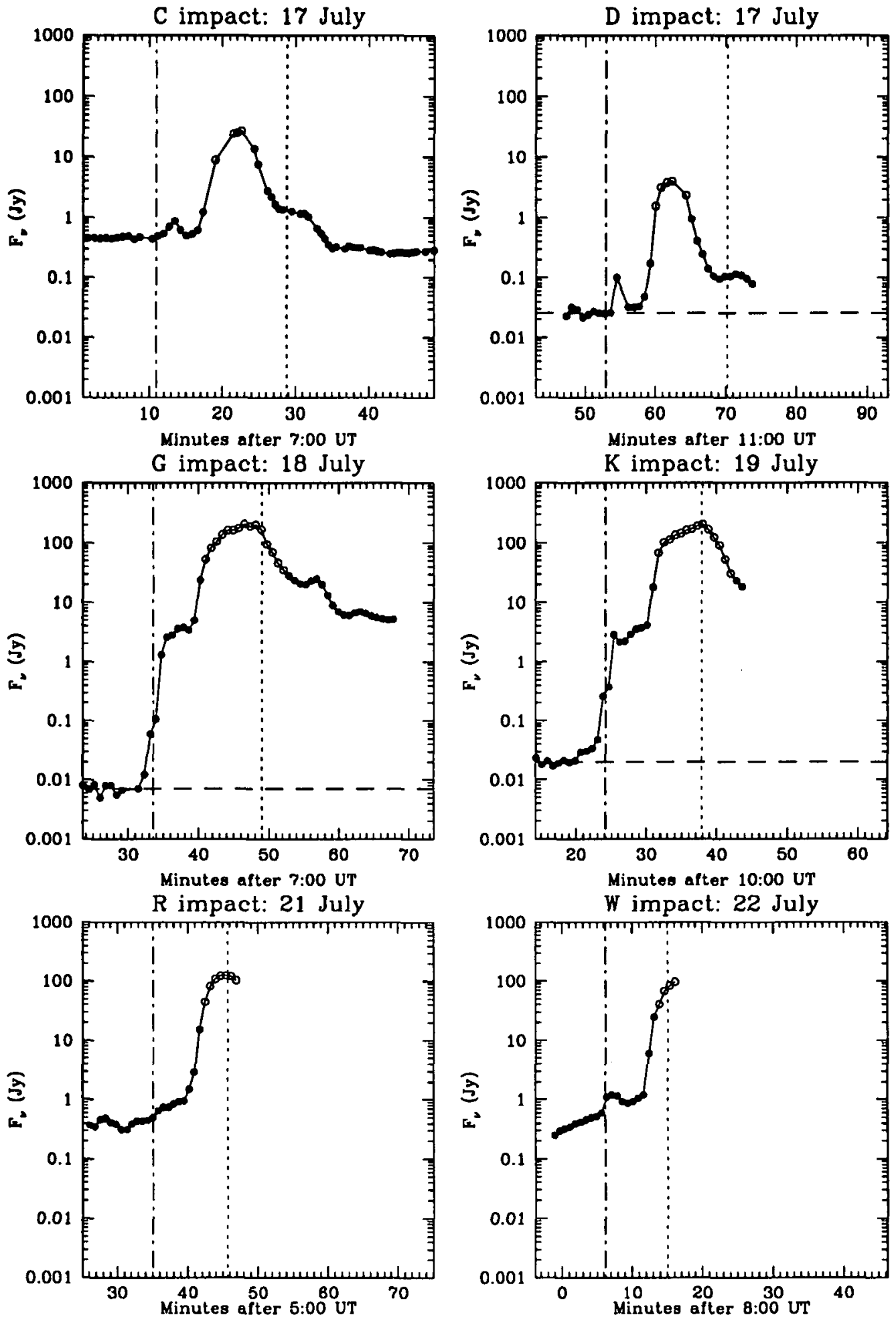

Figure 3. Lightcurves at $2.34 \mu \mathrm{m}$ obtained at Siding Spring Observatory, Australia. Open symbols indicate frames in which the brightest part of the image exceeded the linear range of the detector, and the total flux is likely to be underestimated. Vertical dot-dashed lines indicate the adopted impact times from Table 1, while vertical dashed lines indicate the time at which the impact site reached the planet's limb (see Fig. 2). From McGregor et al. (1995). 

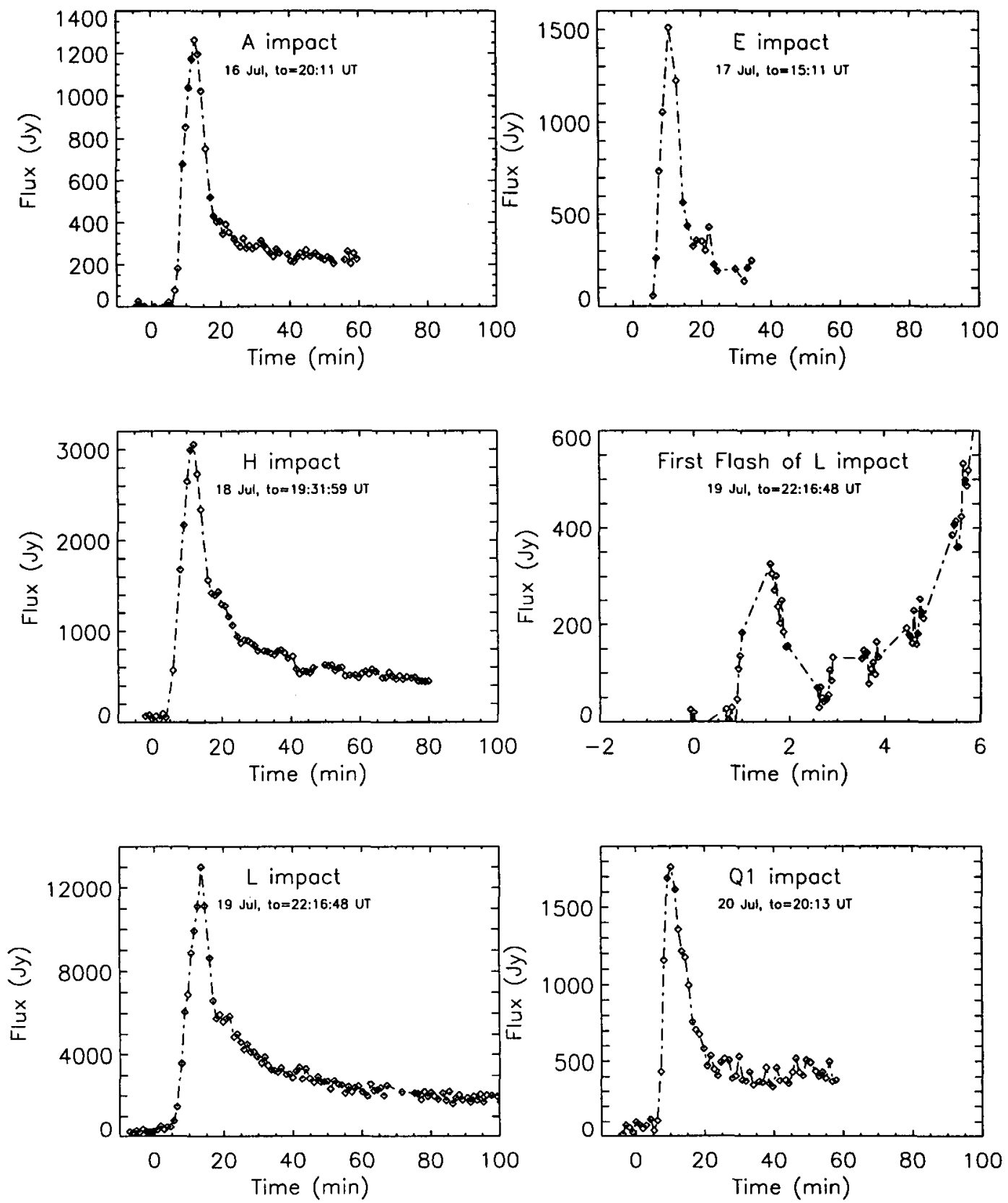

Figure 4. Lightcurves at $12 \mu \mathrm{m}$ obtained at La Palma in the Canary Islands. The abscissa is time from the nominal impact time, as indicated in each panel. From Lagage et al. (1995).

(i) Large impacts G, K and L produced peak IR fluxes of 200 Jy or greater, and were associated with prominent post-impact sites visible in both the HST images and in Earth-based near-IR images.

(ii) Medium-sized impacts (peak fluxes in the range 50-200 Jy) include $\mathrm{E}, \mathrm{H}, \mathrm{Q} 1, \mathrm{R}$, $S$ and $W$. All except $S$ and $W$ (which landed very close to the already complex $G$ and $\mathrm{K}$ sites and were difficult to distinguish as a result) produced post-impact features of class 2 in the HST images, which were also readily detectable in Earth-based images. 


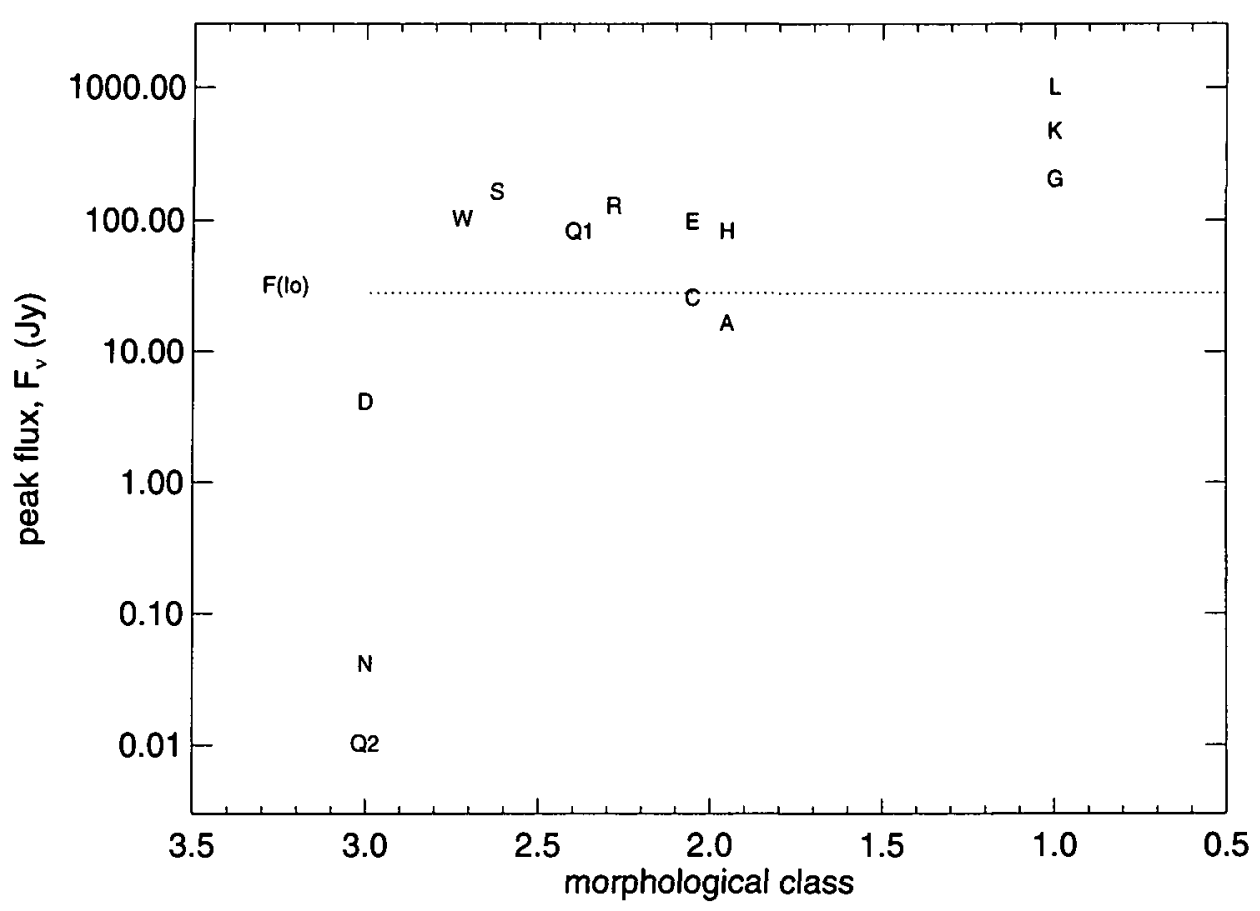

FIGURE 5. Comparison of main-event peak flux at $2.3 \mu \mathrm{m}$ vs. impact classification based on HST images by Hammel et al. (1995). Near-IR data from various sources. In some cases approximate corrections have been made to allow for detector saturation.

(iii) Impacts A, C and D we classify as small, due to their smaller peak near-IR fluxes of 5-20 Jy, and to the comparative faintness of the impact sites in Earth-based nearIR images. The $D$ site was, in fact, indistinguishable from the nearby $G$ site in most ground-based images. However, both $\mathrm{A}$ and $\mathrm{C}$ were rated as class $2 \mathrm{a}$ on the basis of HST images, and the peak $12 \mu \mathrm{m}$ flux from the $\mathrm{A}$ impact was comparable to that seen for $\mathrm{E}$ and Q1 (cf. Fig. 4).

(iv) Impacts B, M, N, Q2, U and V we classify as 'wimps', due to very faint and/or uncertain detections of the impacts themselves. For none of these impacts was a remnant detectable in Earth-based near-IR images of Jupiter, and only sites B, N and Q2 were detectable in the higher-resolution HST images. Impacts B and M were detected only by the Keck telescope. Q2 produced an obvious precursor, but a very weak main event. V appears to have been unique in that a precursor event was observed at two stations, but with no subsequent main event. Impact $U$ is questionable, given at least two negative reports from larger telescopes. See $\S 5$ for further details on these events.

(v) No credible reports of impact signatures exist for fragments F, G2, J, P1, P2 and $\mathrm{T}$, and there are no identifiable impact sites corresponding to the predicted impact locations in the HST images. Fragments $\mathrm{J}$ and $\mathrm{M}$ had in fact disappeared at least six months prior to July 1994, while fragments P1 and P2 had been observed to be further disintegrating (Weaver et al. 1995).

It is also of interest to compare the luminosities of the impact events with the relative sizes of the fragments inferred from their brightness in pre-impact HST images (Weaver et al. 1995). In Fig. 6 we make such a comparison, again using the peak $2.3 \mu \mathrm{m}$ flux as a yardstick. (A'Hearn et al. (1995) present a similar plot using peak and integrated Galileo fluxes at $0.945 \mu \mathrm{m}$.) On average, the peak luminosity is roughly proportional to the cube of the estimated pre-impact diameter, i.e., to the putative mass and ki- 


\begin{tabular}{|c|c|c|c|c|c|c|c|c|}
\hline Fragment & Date/time (UT) & PC1 & $\mathrm{PC} 2$ & $\mathrm{ME}$ & Spot & Ejecta & HST & Galileo \\
\hline A & July $16,20: 12$ & - & $\mathrm{Y}$ & $Y$ & $\mathrm{Y}$ & $\mathrm{Y}$ & $2 \mathrm{a}$ & - \\
\hline B & July $17,02: 50$ & - & - & $?$ & $\mathrm{Y}$ & - & 3 & - \\
\hline $\mathrm{C}$ & July $17,07: 11$ & - & $\mathrm{Y}$ & $\mathrm{Y}$ & $Y$ & $\mathrm{Y}$ & $2 a$ & - \\
\hline $\mathrm{D}$ & July $17,11: 53$ & - & $\mathrm{Y}$ & $\mathrm{Y}$ & $\mathrm{Y}$ & - & 3 & - \\
\hline $\mathrm{E}$ & July $17,15: 12$ & - & - & $\mathrm{Y}$ & $\mathrm{Y}$ & $\mathrm{Y}$ & $2 a$ & - \\
\hline $\mathrm{F}$ & July $18,00: 37$ & - & - & - & - & - & - & - \\
\hline $\mathrm{G}$ & July $18,07: 33: 32$ & $\mathrm{Y}$ & $\mathbf{Y}$ & $Y$ & $Y$ & $\mathrm{Y}$ & 1 & UVS/PPR/NIMS \\
\hline $\mathrm{H}$ & July $18,19: 31: 59$ & $\mathrm{Y}$ & $\mathrm{Y}$ & $\mathrm{Y}$ & $\mathrm{Y}$ & $\mathrm{Y}$ & $2 a$ & PPR \\
\hline $\mathrm{J}$ & July $19,01: 35$ & - & - & - & - & - & - & - \\
\hline $\mathrm{K}$ & July $19,10: 24: 13$ & $\mathrm{Y}$ & $Y$ & $\mathrm{Y}$ & $\mathrm{Y}$ & $\mathrm{Y}$ & 1 & SSI \\
\hline $\mathrm{L}$ & July $19,22: 16: 48$ & $\mathrm{Y}$ & $\mathrm{Y}$ & $\mathrm{Y}$ & $\mathrm{Y}$ & $\mathrm{Y}$ & 1 & PPR \\
\hline $\mathrm{M}$ & July $20,06: 02$ & - & - & $?$ & - & - & - & - \\
\hline $\mathrm{N}$ & July $20,10: 29: 17$ & - & - & Y & $\mathrm{Y}$ & - & 3 & SSI \\
\hline P2 & July $20,15: 23$ & - & - & - & - & - & - & - \\
\hline $\mathrm{P} 1$ & July $20,16: 37$ & - & _- & _- & _- & _- & _ & - \\
\hline Q2 & July $20,19: 44$ & - & $\mathrm{Y}$ & $\mathrm{Y}$ & $\mathrm{Y}$ & - & 3 & - \\
\hline Q1 & July $20,20: 13: 52$ & - & $\mathrm{Y}$ & $\mathrm{Y}$ & $\mathrm{Y}$ & $\mathrm{Y}$ & $2 b$ & PPR \\
\hline $\mathrm{R}$ & July $21,05: 35: 03$ & $\mathrm{Y}$ & $\mathrm{Y}$ & $\mathrm{Y}$ & $\mathrm{Y}$ & $\mathrm{Y}$ & $2 \mathrm{~b}$ & NIMS \\
\hline $\mathrm{S}$ & July $21,15: 16$ & - & $\mathrm{Y}$ & $\mathrm{Y}$ & $\mathrm{Y}$ & $?$ & $2 c$ & - \\
\hline $\mathrm{T}$ & July $21,18: 11$ & _- & - & - & - & - & - & - \\
\hline $\mathrm{U}$ & July $21,21: 56$ & - & - & $?$ & - & - & - & - \\
\hline $\mathrm{V}$ & July $22,04: 23: 10$ & $Y ?$ & - & - & _- & - & _- & - \\
\hline $\mathrm{W}$ & July $22,08: 06: 14$ & - & $\mathrm{Y}$ & $\mathrm{Y}$ & $\mathrm{Y}$ & $?$ & $2 c$ & SSI \\
\hline
\end{tabular}

TABLE 1. Summary of impact times, observed phenomena, HST impact site classifications and availability of Galileo data for the individual SL9 fragments. PC1 and PC2 refer to Earth-based detections of the first and second precursors, $M E$ to observations of the infrared main event, and 'spot' and 'ejecta' to the visibility of central spots and crescent-shaped ejecta blankets in the Hubble Space Telescope (HST) images. Question marks refer to uncertain observations, or to the uncertain interpretation of the $\mathrm{V}$ event. Galileo instruments are indicated by their standard abbreviations.

netic energy of the fragment, as might be expected. The correlation between fragment size (inferred from their relative brightnesses) and impact flux is, however, by no means a one-to-one relationship. Although the brightest IR signals were associated with the large $\mathrm{G}, \mathrm{K}$ and $\mathrm{L}$ fragments, the impact of the equally-bright $\mathrm{Q} 1$ produced an unexpectedly faint infrared signature-and a correspondingly average-sized impact scar on the planet-while the slightly fainter Q2 fragment yielded one of the weakest main events detected. Fragments F, P2, and B might have been expected to produce signatures at least comparable to those readily detected from the smaller A and D fragments.

More significant perhaps is the observation by Weaver et al. (1995) that the fragments located off the line of the main SL9 train produced smaller ejecta patterns than their brightnesses would have predicted. These include fragments B, F, G2, N, P2, P1, Q2, $\mathrm{T}, \mathrm{U}$ and $\mathrm{V}$. If the vanished $\mathrm{J}$ and $\mathrm{M}$ fragments are included, this list is identical to that of our categories (iv) and (v) above. It seems likely that these fragments were of lower density and much less cohesive than their siblings, perhaps being no more than weakly-bound clumps of debris which entered the jovian atmosphere as meteor showers rather than as coherent bolides. 


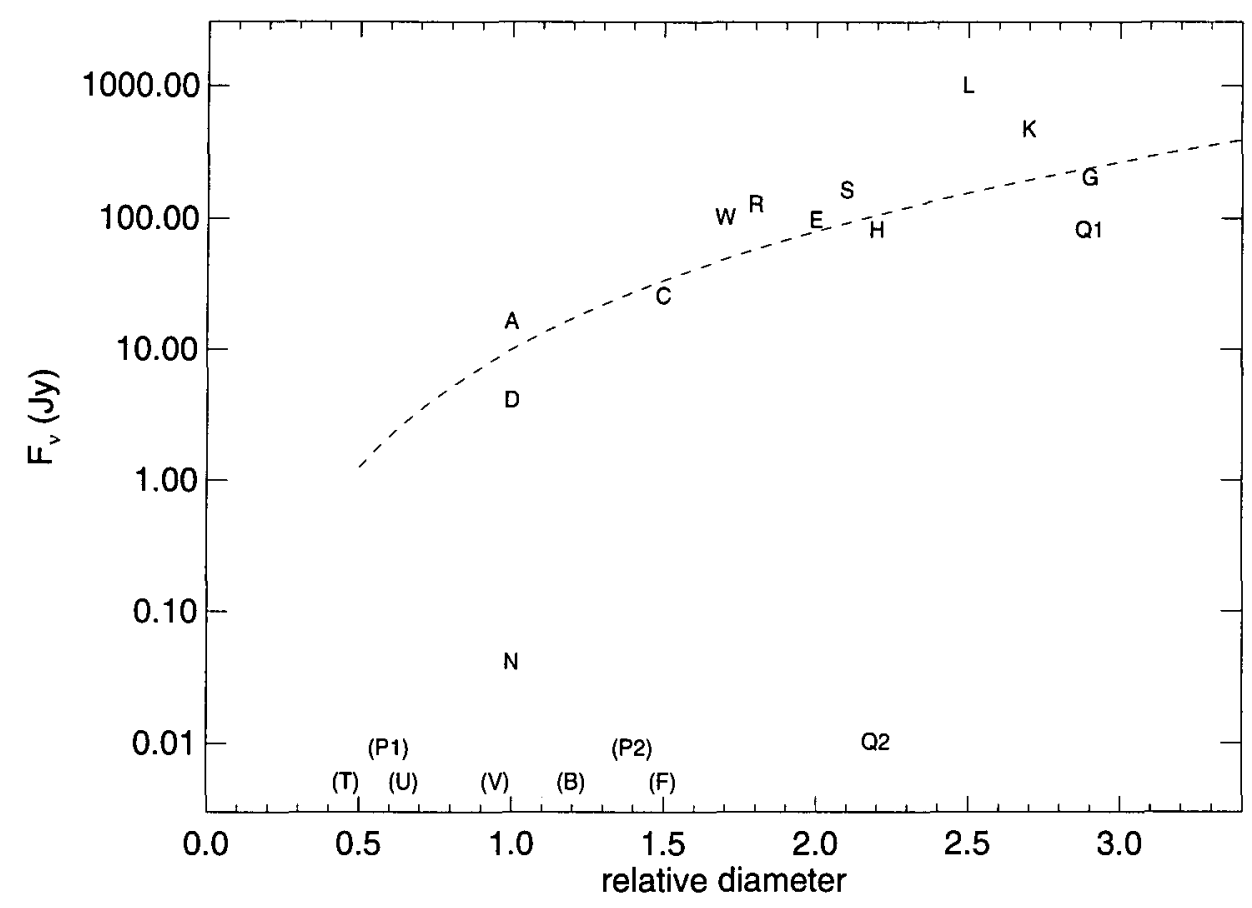

FIGURE 6. Comparison of main-event peak flux at $2.3 \mu \mathrm{m}$ vs. pre-impact relative fragment sizes derived from HST images by Weaver et al. (1995). Near-IR data as in Fig. 5. Labels in parentheses indicate either non-detections or very weak detections. The dashed curve is a simple cubic fit.

\section{Anatomy of a lightcurve}

The various phases in the impact lightcurves are seen most clearly in the $\mathrm{R}$ lightcurves in Fig. 1 and in the data from the well-observed G, H, K and L impacts. These lightcurves show very similar characteristics, and we are fortunate in having Galileo observations for all five events, as well as a series of HST images for the G impact. Figures 7 and 8 show the early phases of the $\mathrm{G}$ and $\mathrm{K}$ impacts, using $2.3 \mu \mathrm{m}$ lightcurves from Siding Spring (McGregor et al. 1995) and the Okayama Astrophysical Observatory, Japan (Watanabe et al. 1995; Takeuchi et al. 1995). In Fig. 7 the periods during which the Galileo PPR, UVS and NIMS instruments observed emission are indicated (Martin et al. 1995; Hord et al. 1995; Carlson et al. 1995a), as are the exposure times of individual HST images (Hammel et al. 1995). The period during which the Galileo SSI instrument observed the flash from the $\mathrm{K}$ impact (Chapman et al. 1995) is shown in Fig. 8.

The early phases of the $\mathrm{H}$ and $\mathrm{L}$ impacts-- the latter being arguably the most energetic of all the SL9 events-are shown in Fig. 9, with lightcurves from the 2.2 and $3.5 \mathrm{~m}$ telescopes at Calar Alto, Spain (Hamilton et al. 1995) and the $1 \mathrm{~m}$ telescope at Pic du Midi, France (Colas et al. 1995; Drossart et al. 1995). For L, Fig. 9(a) shows a very abrupt first precursor at 22:16:41 UT, followed by a decay over the next 50 sec until the onset of the second precursor at 22:17:30 UT saturated the detector. The Pic du Midi observations in Fig. 9(b) carry the L lightcurve through the second precursor phase, until saturation occurred at the beginning of the main event at about 22:23 UT.

\subsection{A simple ballistic model}

Although a full understanding of the process whereby the kinetic energy of the incoming fragments of SL9 produced the spectacular events seen by Galileo, HST and Earth-based 


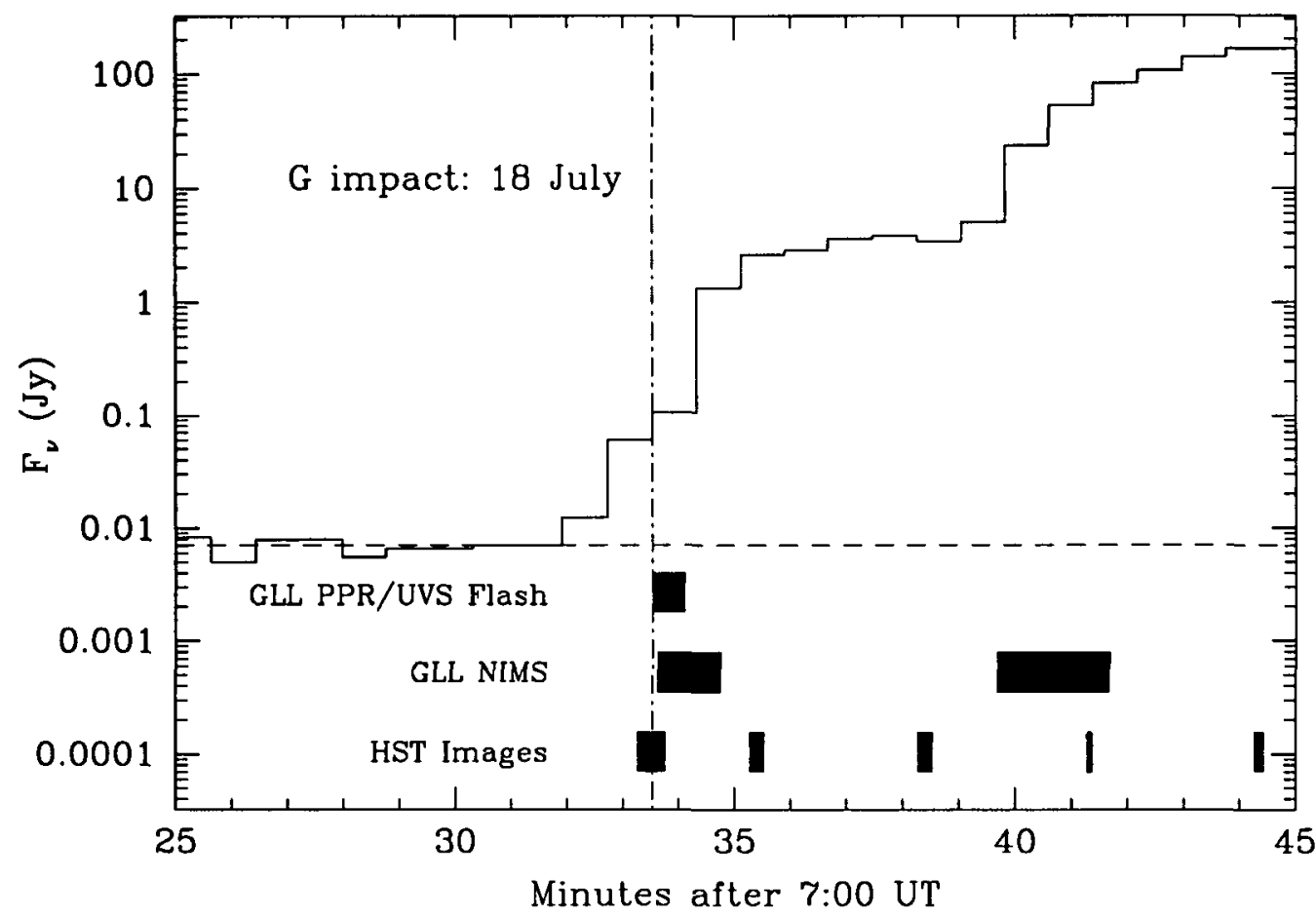

Figure 7. Lightcurve for the G impact obtained at Siding Spring Observatory, Australia at $2.34 \mu \mathrm{m}$. Steps indicate individual $30 \mathrm{sec}$ exposures at $\sim 50 \mathrm{sec}$ intervals. A vertical dot-dashed line indicates the adopted impact time, as set by the onset of the Galileo PPR/UVS flash. Blocks indicate periods of emission observed by Galileo, and exposure times for HST images. From McGregor et al. (1995).

telescopes depends on detailed numerical modelling of the 3-D hydrodynamics of the impact (cf. the chapter by Crawford in this volume), the subsequent evolution of the plumes themselves was largely governed by simple ballistics (see chapters by Zahnle and MacLow). Guided by the observations of maximum plume height and debris distribution by HST (Hammel et al. 1995), and the conceptual models of Boslough et al. (1995) and Zahnle and MacLow (1995), we can construct a purely ballistic model which accounts for the timing of most of the features seen in the SL9 lightcurves (cf. also Takeuchi et al. 1995; Drossart et al. 1995).

A maximum vertical ejection velocity for the impact debris cloud of $v_{z} \simeq 12.5 \mathrm{~km} \mathrm{~s}^{-1}$ is set by the observed maximum plume height of $v_{z}^{2} / 2 g=3200 \mathrm{~km}$ (Hammel et al. 1995). The $12,500 \mathrm{~km}$ outer radius of the 'crescent' of debris seen around the G impact site in HST images (West et al. 1995) suggests a maximum ejection velocity of $v_{e}=\sqrt{g r} \simeq$ $18 \mathrm{~km} \mathrm{~s}^{-1}$, although Zahnle has argued that the crescent may have expanded significantly after re-entry of the plume material. Both results are consistent with a relatively flat plume trajectory, with a maximum elevation angle $\theta_{\max } \simeq 45^{\circ}$. An ejection angle of $45^{\circ}$ would also match the incoming trajectory of the fragment relative to Jupiter's cloud tops, back along which the bulk of ejecta is predicted to have been ejected (Boslough et al. 1995). In reality, of course, the ejecta emerged with a distribution of initial velocities and/or directions, as demonstrated by the broad crescent-shaped ejecta patterns, although the asymmetry of the ejecta blankets seen in the HST images confirms that the plume material was primarily ejected back along the fragments' inbound trajec- 


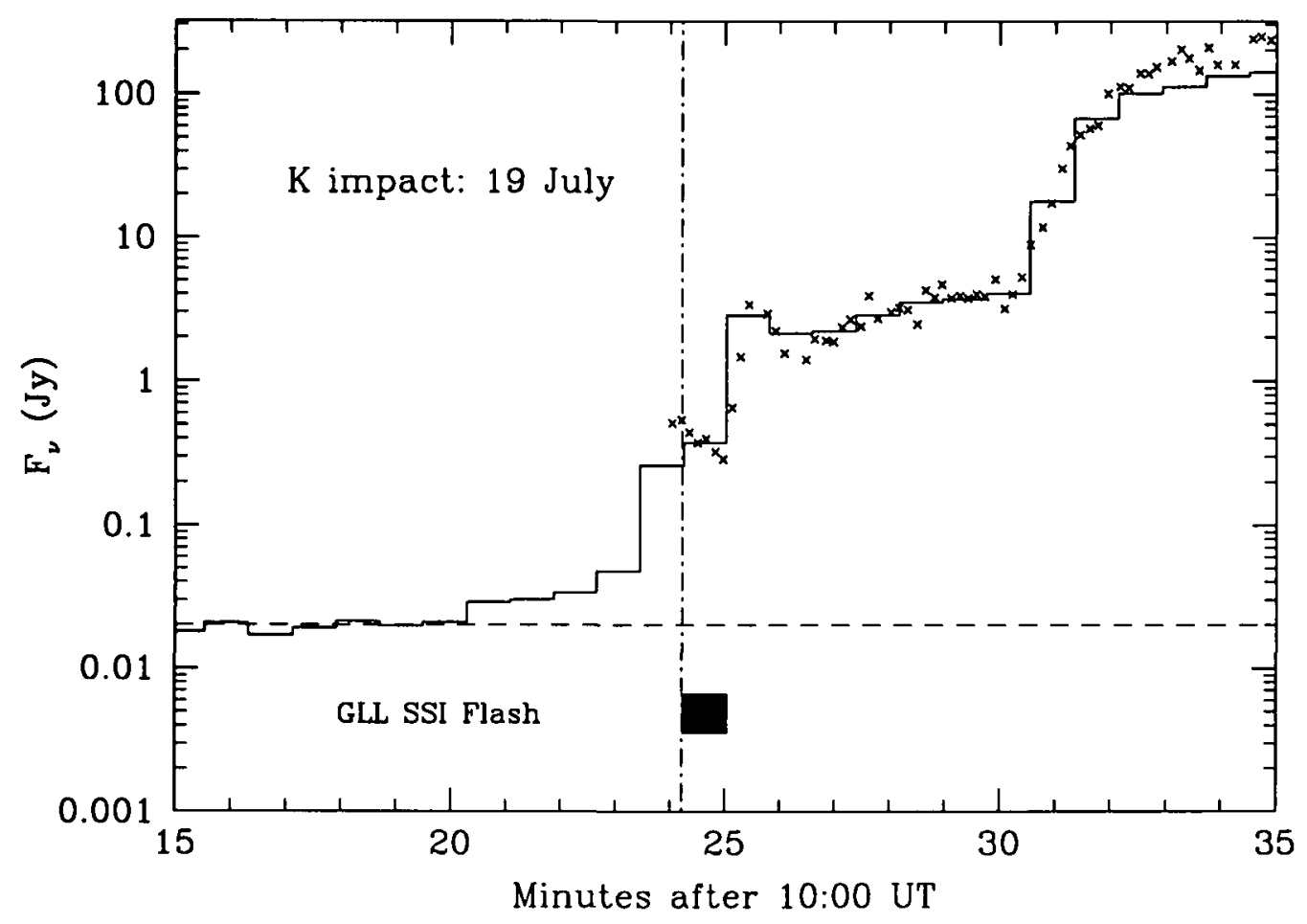

FIGURE 8. Lightcurves for the $\mathrm{K}$ impact obtained at Siding Spring Observatory, Australia at $2.34 \mu \mathrm{m}$ (histogram) and the Okayama Astrophysical Observatory, Japan at $2.35 \mu \mathrm{m}$ (crosses). The OAO data are $1 \mathrm{sec}$ exposures taken with an IR camera and narrowband $2.35 \mu \mathrm{m}$ filter, at $10 \mathrm{sec}$ intervals. Neutral density filters were used during the period of peak brightness to avoid detector saturation. A vertical dot-dashed line indicates the adopted impact time, as set by the onset of the Galileo SSI flash. From McGregor et al. (1995).

tory, or towards the south-east once allowance is made for Coriolis deflection (Hammel et al. 1995).

Figure 10 shows sample trajectories for plume tracers ejected at a common velocity of $18 \mathrm{~km} \mathrm{~s}^{-1}$ and at elevation angles of $15^{\circ}, 30^{\circ}$ and $45^{\circ}$ from the horizontal. Superimposed on the particle trajectories are a set of curves indicating the time and altitude at which the ejecta rises above the planet's limb into the view of Earth-based observers, for an ejection azimuth $45^{\circ}$ east of south. (These curves also depend weakly on the assumed elevation angle of the ejecta, as they are controlled by both the planet's rotation and the horizontal velocity of the plume: the plotted curves are for $\theta=30^{\circ}$.) The different curves for different impacts reflect the varying location of the impact points behind the limb; later impacts generally falling closer to the limb (Chodas \& Yeomans, this volume). The $\mathrm{G}$ plume, for example, would have become directly visible 32 sec after impact for $\theta=45^{\circ}$, whereas the $\mathrm{W}$ plume emerged into view after only $12 \mathrm{sec}$.

The dashed curves in Fig. 10 indicate the time and altitude at which the ejecta rose above the shadow of the jovian limb, and into direct sunlight. Again, the plumes from the later impacts, starting closer to the terminator, rise into sunlight earlier and at a lower altitude. For G, the plume remains in shadow until $130 \mathrm{sec}$ after impact and $1500 \mathrm{~km}$ altitude, while for $\mathrm{W}$ the interval is only $90 \mathrm{sec}$ and the corresponding altitude $\sim 1000 \mathrm{~km}$, all for $\theta=45^{\circ}$. These times are consistent with the sequences of images obtained for these two impacts by HST (Hammel et al. 1995): the G plume is seen in 

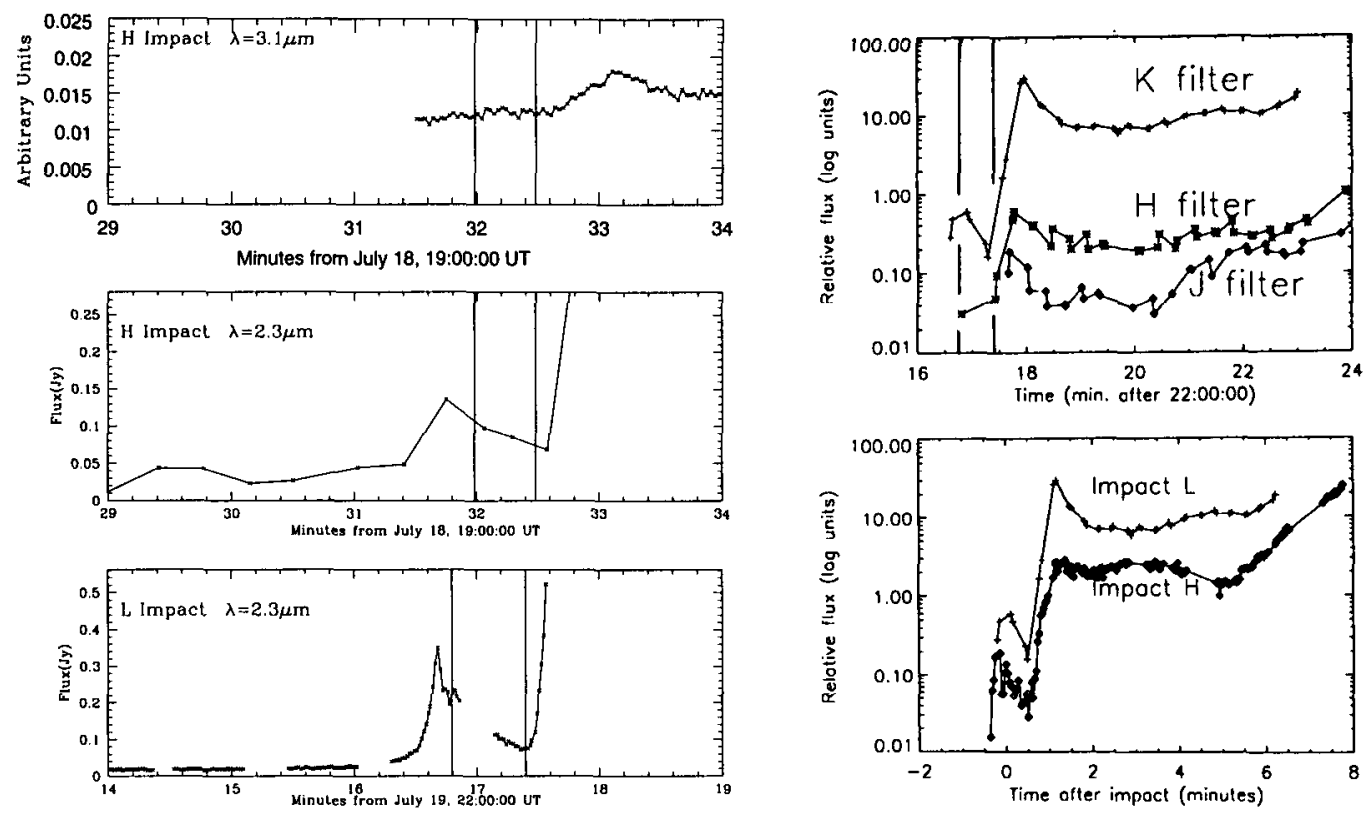

Figure 9. (a) Precursor lightcurves for the $\mathrm{H}$ and $\mathrm{L}$ impacts obtained at the 2.2 and $3.5 \mathrm{~m}$ telescopes at Calar Alto Observatory, Spain. The $2.30 \mu \mathrm{m}$ data were obtained with an IR camera and a narrowband methane filter, while the $3.1 \mu \mathrm{m}$ data were obtained with a high-speed photometer. For L the time resolution was $1.2 \mathrm{sec}$. From Hamilton et al. (1995). (b) Lightcurves for the $\mathrm{H}$ and $\mathrm{L}$ precursors obtained at the $1 \mathrm{~m}$ telescope at Pic du Midi Observatory, France. The data were obtained with a multi-channel IR camera and standard $J, H$ and $K^{\prime}(1.25,1.65$ and $2.12 \mu \mathrm{m}$ ) filters, at $\sim 18 \mathrm{sec}$ intervals. The lower panel compares the $2.12 \mu \mathrm{m}$ data on a common scale. From Colas et al. (1995).

thermal emission at $T_{i}+2.0 \mathrm{~min}$ and in sunlight at $T_{i}+5.0 \mathrm{~min}$; the $\mathrm{W}$ plume is seen emerging into sunlight at $T_{i}+3.0$ min.

We now discuss the successive phases in a typical SL9 lightcurve, with this highly simplified but illustrative model as a guide to their interpretation.

\subsection{Leader emission}

For some minutes prior to the actual impact time, faint emission is seen in the $G, K$ and $L$ lightcurves. This leader emission brightens gradually, and then more steeply, until it merges into the first precursor flash. Its duration ranges from $\sim 30 \mathrm{sec}$ for L (Fig. 9) to 3.5 min for K (Fig. 8), and is far too long to be accounted for by the passage of the main fragment through Jupiter's atmosphere at the entry velocity of $60 \mathrm{~km} \mathrm{~s}^{-1}$. More likely, this emission is due to an extended stream of dust preceding the main fragment, which arrived as a 'meteor shower' in Jupiter's atmosphere (Meadows et al. 1995; McGregor et al. 1995). The durations of the leaders are consistent with the elongations of the cometary comae over several tens of thousands of $\mathrm{km}$ observed prior to impact (Weaver et al. 1995). For the $\mathrm{L}$ event, a fainter feature was visible on the limb for at least 20 min prior to the impact, but it is not clear if this also represents 'leader' emission or 


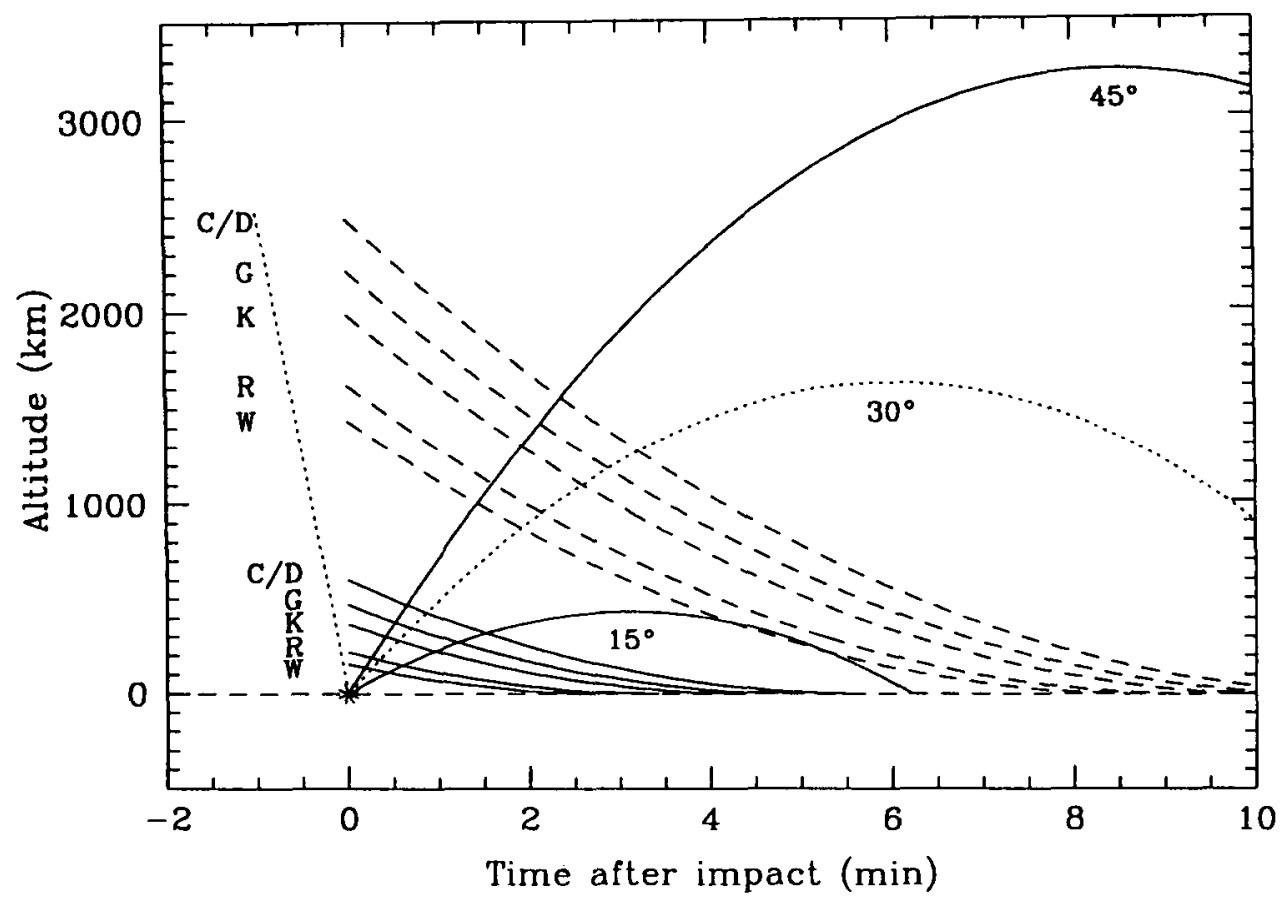

FIGURE 10. Plume altitude vs. time for an ejection velocity $v_{e}=18 \mathrm{~km} \mathrm{~s}^{-1}$ and ejection angles $\theta=15^{\circ}, 30^{\circ}$ and $45^{\circ}$. Solid sloping lines indicate the altitude at which plume material becomes directly visible to an earth-based observer, for representative impacts, while the dashed lines indicate where the plume enters sunlight for the same impacts. The trajectory of the incoming fragments is shown by the dotted line at negative time. From McGregor et al. (1995).

the remnant of an earlier impact by an uncatalogued or lost (J?) fragment (Hamilton et al. 1995).

\subsection{First precursor}

First precursors have been identified convincingly only in the lightcurves for the $G, H$, $\mathrm{K}, \mathrm{L}$ and $\mathrm{R}$ impacts, with the highest resolution data being available for the $\mathrm{R}, \mathrm{K}$ and L events (Figs. 1, 8 \& 9(a)). The first $\mathrm{L}$ precursor peaked sharply $7 \mathrm{sec}$ prior to the initial Galileo PPR detection, declined to a plateau for about $8 \mathrm{sec}$, and then faded gradually over the next $40 \mathrm{sec}$ (Hamilton et al. 1995). A similar pattern was observed for $\mathrm{K}$, where the first precursor appeared abruptly and peaked 11 sec prior to the beginning of the flash observed by the Galileo SSI instrument (Watanabe et al. 1995), before decaying slowly over the next $60 \mathrm{sec}$. The first $\mathrm{R}$ precursor shows a similar development at $2.3 \mu \mathrm{m}$, beginning abruptly $\sim 24$ sec prior to the Galileo NIMS flash, and then decaying slowly (Graham et al. 1995). The Siding Spring lightcurve for G shows but does not fully resolve the first precursor, which appears only as a 2-frame pause before it is overtaken by the second precursor (McGregor et, al. 1995). In Calar Alto observations of the H impact, the first precursor appears in only four frames (Hamilton et al. 1995). For both G and $\mathrm{H}$ the initial abrupt flux increase occurs in the image obtained immediately prior to the onset of the corresponding Galileo flash, consistent with the higher-resolution $\mathrm{K}, \mathrm{L}$ and $\mathrm{R}$ data.

The timing of the first precursor, as well as the steeply-brightening leader seen for $\mathrm{L}$, strongly suggests that it was due to thermal emission from the main fragment as it entered Jupiter's upper atmosphere, $400-650 \mathrm{~km}$ above the effective impact level. Inspection of 
Fig. 10 shows that the $\mathrm{K}$ and $\mathrm{L}$ meteors were indeed last directly visible above the limb at about this altitude. The $\sim 10 \mathrm{sec}$ gap between the first precursor peak and the onset of the Galileo flash represents the flight time of the bolide behind the jovian limb. The slow decay of the first precursor has been attributed to cooling of the heated trail left by the entering body (Hamilton et al. 1995; McGregor et al. 1995), while it has been suggested (Chapman 1995, private communication) that the plateau in the L data may be due to reflection of the initial fireball from infalling dust high in the atmosphere.

Finally, we note that a $30 \mathrm{sec}$ exposure of the G impact taken by HST centered on the start time of the Galileo PPR/UVS flash (cf. Fig. 7 and Hammel et al. 1995) shows a point-like source of emission in the planet's shadow. Since the rising plume cannot have been visible at the Earth within $15 \mathrm{sec}$ after the impact (see Fig. 10), this frame must also have captured the meteor during its passage through the upper atmosphere $\sim 10 \mathrm{sec}$ prior to impact.

\subsection{Second precursor}

Approximately 55-60 sec after the onset of the first precursor, a much brighter second precursor was seen for each of the larger impacts (cf. Figs. 1(b), 7, 8 \& 9). In the case of the less energetic impacts such as A, C, D, Q2, Q1, S and W, only a single precursor was detected; we assume that this corresponds to the second precursor, although for the late W impact both precursors may have merged into a single event (McGregor et al. 1995). The rise time of the second precursor was 15-20 sec, and the duration (FWHM) was typically 30-60 sec. For the weaker impacts (e.g., C, D, Q2, Q1 and W), the flux soon thereafter returned to the pre-impact level (cf. Fig. 3 and Herbst et al. 1995, Fig. 1).

For the bright G, H, K and L impacts, on the other hand, the flux level after the second precursor dipped only slightly and then remained relatively constant-or even increased slowly - for $\sim 4$ min until the main event began. The $\mathrm{R}$ impact presents a particularly interesting case, with the fluxes at 3.2 and $4.5 \mu \mathrm{m}$ fading quite rapidly after the second precursor peaked, while the $2.3 \mu \mathrm{m}$ flux declines only slowly (cf. Fig. 1(b)). A similar wavelength-dependence is shown by the $\mathrm{H}$ impact, where the flux was observed to drop more sharply after the second precursor at $3.1 \mu \mathrm{m}$ than at $2.3 \mu \mathrm{m}$ (Hamilton et al. 1995). Mid-IR observations of the $\mathrm{H}$ and $\mathrm{L}$ impacts show second precursors for these events at 10-12 $\mu \mathrm{m}$, which also faded after 30-60 sec (Livengood et al. 1994; Lagage et al. 1995) in contrast to their persistence at $2.3 \mu \mathrm{m}$.

There is some indication that the time of peak flux for the second precursor is also wavelength-dependent, although results are inconsistent. Hamilton et al. (1995) found that the $\mathrm{H}$ precursor peaked $10-20 \mathrm{sec}$ earlier at $3.1 \mu \mathrm{m}$ than at $2.3 \mu \mathrm{m}$. Nicholson et al. (1995a) similarly noted that the $\mathrm{R}$ precursor began $\sim 20$ sec earlier at $4.5 \mu \mathrm{m}$ than at $2.3 \mu \mathrm{m}$. However, Colas et al. (1995) find that the L precursor peaked $\sim 15 \mathrm{sec}$ later at $2.12 \mu \mathrm{m}$ than at 1.25 or $1.65 \mu \mathrm{m}$. Drossart et al. (1995) and Lagage et al. (1995) note a $30 \mathrm{sec}$ delay between the onset of the second $\mathrm{L}$ precursor at $2.3 \mu \mathrm{m}$ and at $12 \mu \mathrm{m}$, although the second $H$ precursor seems to have been seen essentially simultaneously at $2.3 \mu \mathrm{m}$ and at $10 \mu \mathrm{m}$ (Livengood et al. 1994). No obvious pattern emerges from these reports.

The second precursor has generally been interpreted as the first appearance of the fireball as seen from the Earth, rising above the jovian limb (e.g., Graham et al. 1995). Comparison of the reported times for the second precursor with those of the initial Galileo flashes show that the $2.3 \mu \mathrm{m}$ second precursors began $70 \pm 18 \mathrm{sec}(\mathrm{G}), 48 \pm 12 \mathrm{sec}(\mathrm{H})$, $50 \pm 7 \mathrm{sec}(\mathrm{K}), 39 \pm 4 \mathrm{sec}(\mathrm{L}), 38 \pm 11 \mathrm{sec}(\mathrm{R})$ and $10 \pm 16 \mathrm{sec}(\mathrm{W})$ after the actual impact times (see chapter by Chodas \& Yeomans). This pattern of decreasing intervals is consistent with the progressively shorter times taken by the rising fireball to reach an 
altitude at which it is directly visible from earth, as illustrated in Fig. 10. The actual intervals between the impact flash and the arrival of the fireball at the limb also depend on jovian atmospheric extinction. Estimates of the pressure level at which the line-of-sight optical depth is of order unity range from $\sim 15 \mathrm{mb}$ at 2.3 or $3.5 \mu \mathrm{m}$ (Meadows et al. 1995) to $100 \mathrm{mb}$ at $10 \mu \mathrm{m}$ (Livengood et al. 1995). These levels are, respectively, $90 \mathrm{~km}$ and $45 \mathrm{~km}$ above the $1 \mathrm{bar}$ level, the approximate depth at which the explosion is believed to have been initiated (Boslough et al. 1995; Zahnle and MacLow 1995). Assuming a maximum vertical velocity of $12.5 \mathrm{~km} \mathrm{~s}^{-1}$, and neglecting the initial acceleration phase of the explosion, we find that the $\mathrm{G}$ and $\mathrm{H}$ fireballs should have become visible at $2.3 \mu \mathrm{m}$ no sooner than $40 \mathrm{sec}$ after impact, while the $\mathrm{R}$ and $\mathrm{W}$ fireballs should have appeared after about 24 and $20 \mathrm{sec}$, respectively. These predictions are in reasonable agreement with the observed intervals quoted above.

This interpretation is strengthened by Galileo NIMS observations of the G impact, which clearly show a rising, cooling fireball at the same time as the second precursor was beginning (cf. Fig. 7). An HST image taken immediately after the Galileo flash had faded (at 7:35:16 UT) shows thermal emission in the planet's shadow from the rising plume. Near-IR spectra taken during the second precursor phase of the K impact show a blue continuum during the first minute of the second precursor, which then rapidly cooled over the succeeding few minutes (Meadows \& Crisp 1995)-again consistent with a rising fireball.

The absence of any noticeable increase in brightness in the second precursors $\sim 2 \mathrm{~min}$ after impact as the plumes crossed the terminator into sunlight (cf. Fig. 10) argues against any appreciable fraction of the near-IR flux at this time being due to reflected sunlight from condensates which may have formed in the plume.

In the context of a rising, cooling plume, the flat or slowly increasing flux level exhibited by the G, H, K and L precursors at $2.3 \mu \mathrm{m}$ is puzzling. McGregor et al. (1995) have suggested that this may be due to the continued ejection of hot material from the entry site after the initial fireball for the most energetic (and deepest penetrating?) impacts. Some support for this explanation is provided by an HST image of the G plume taken at 7:38:16 UT, almost $5 \mathrm{~min}$ after the impact. This image appears to show the plume in sunlight, but with a faint 'tail' of thermal emission in the shadow region (Hammel et al. 1995). This tail could be interpreted in terms of continued ejection along the entry corridor. It is not clear, however, how the more rapid decay of the second precursors at $3.1 \mu \mathrm{m}$ and longer wavelengths is to be interpreted in this context, as an increase in ejecta temperature over time seems rather unlikely. A similar problem arises in connection with the slow decay of the second $\mathrm{R}$ precursor at $2.3 \mu \mathrm{m}$.

\subsection{Main event}

Approximately $6 \mathrm{~min}$ after each recorded impact, a steady and in some cases truly awesome brightening was observed at near-IR and mid-IR wavelengths. In the case of some of the smaller impacts, or when observing conditions were below par, this main event was the only feature observed. An initially rapid rise over 1-2 min was followed by a more gradual increase, the intensity reaching a peak 10-15 min after the moment of impact. The interval between impact and peak luminosity was correlated with the peak flux, the brighter events taking longer to reach maximum (Meadows et al. 1995); the average interval was $12 \mathrm{~min}$. The initial decline in intensity following the peak had an e-folding time of $\sim 3 \mathrm{~min}$ at $2.3 \mu \mathrm{m}$, but was somewhat slower at $10-12 \mu \mathrm{m}$ (cf. Figs. 3 and 4). In many cases the decay was interrupted by a plateau or shoulder at $\sim 20 \mathrm{~min}$ after the impact. 
At their peaks, the $\mathrm{G}$ and $\mathrm{K}$ fluxes at $2.3 \mu \mathrm{m}$ probably both reached $\sim 450 \mathrm{Jy}$, or 15 times the brightness of Io, although the $\mathrm{G}$ lightcurve is partly saturated. The peak brightness of the $\mathrm{L}$ impact seems to have been even greater, and this event strongly saturated the array detectors on larger telescopes. Observing at the $0.4 \mathrm{~m}$ telescope at Whately Observatory, Massachusetts, with an IR array and narrowband $2.30 \mu \mathrm{m}$ filter, Skrutskie \& Aas (1995) were able to follow the main L event through only modest saturation to a peak brightness of 35 times that of Io, or $\sim 1000 \mathrm{Jy}$. Very similar main events were observed for impacts at $2.3,3.1-3.2,4.5,10$ and $12 \mu \mathrm{m}$, suggesting that the temperature of the emission remained relatively constant during the entire duration of this phase. At $12 \mu \mathrm{m}$ the peak flux for the $\mathrm{L}$ impact reached $13,000 \mathrm{Jy}$, over 4 times the brightness of the $\mathrm{H}$ event and 8 times that of E and Q1 (cf. Fig. 4).

Although initial discussions of the main event for $R$ (cf. Graham et al. 1995; Nicholson et al. 1995a) emphasized the rough coincidence between the time of peak flux and the time at which the impact site rotated onto the planet's limb, once a larger suite of observations became available it became apparent that this purely geometric interpretation of the lightcurves was not tenable. The extensive series of $2.3 \mu \mathrm{m}$ lightcurves obtained at Siding Spring (McGregor et al. 1995; see Fig. 3), and the similar series of $12 \mu \mathrm{m}$ lightcurves obtained at La Palma (Lagage et al. 1995; see Fig. 4), demonstrate conclusively that the main event began $\sim 6$ min after the impact time in all cases, irrespective of the distance of the impact point behind the limb. In fact, for the early $\mathrm{C}$ and $\mathrm{D}$ impacts, the main event was almost over by the time the impact site had rotated onto the limb (cf. dashed lines in Fig. 3). Moreover, the Galileo NIMS instrument recorded the beginning of the main events for $G$ and $R 370$ sec after the initial flashes (Carlson et al. 1995a; 1995b), at almost exactly the same time as did earth-based observers at $2.3 \mu \mathrm{m}$ (cf. Figs. 1 and 7 ). The average interval between the adopted impact time and the onset of the main event observed in 12 lightcurves (mostly at $2.3 \mu \mathrm{m}$ ) was $5.9 \pm 0.3 \mathrm{~min}$.

It is thus apparent that the origin of the main peak must be sought in the impact phenomenon itself, rather than in the observational geometry. The rapid adiabatic cooling of the rising plume makes it highly unlikely that the plume itself contributes significantly to thermal emission in the near-IR during the main event (Graham et al. 1995; Nicholson et al. 1995a), although this view has been disputed by Meadows et al. (1995) on the basis of spectroscopic evidence. Although several alternative suggestions have been made (e.g., dust formation in the cooling plume-Hasegawa et al. 1995), the most likely explanation is that the tremendous burst of infrared flux is associated with the re-entry of the plume into the jovian atmosphere, where its kinetic energy is converted back to thermal energy via shock-heating (Zahnle \& MacLow 1995; Boslough et. al. 1995)-picturesquely described as the 'splashback' or 'hypervelocity splat'. Note that, because of the range of vertical ejection velocities represented in the plume, there is no contradiction in the lower velocity material beginning its re-entry at about the same time as the highest velocity material is nearing its peak altitude, as seen in the HST plume images (Boslough et al. 1995). Simple models for the infrared luminosity of the re-entering $\mathrm{R}$ impact plume have been generated by Zahnle and MacLow (1995), and are reviewed in the chapter by Zahnle in this volume. These models are quite successful in reproducing both the amplitude and the temporal evolution of the $\mathrm{R}$ main event at $2.3-4.5 \mu \mathrm{m}$, although it should be noted that the opacity source for the shock-heated debris is at present unknown and the model opacity was simply adjusted to fit the observations.

The common interval between impact and onset of the main event can be interpreted as requiring a minimum vertical re-entry velocity in order to generate significant shockheating and/or thermal radiation. A 6 min flight time corresponds to a vertical velocity 
component $v_{z}=g t / 2=4.5 \mathrm{~km} \mathrm{~s}^{-1}$. Zahnle (this volume) has in fact suggested that a minimum vertical re-entry velocity of $4-5 \mathrm{~km} \mathrm{~s}^{-1}$ is needed to heat jovian air sufficiently for shock synthesis to produce abundant organic solids as opacity sources. At lower shock velocities, the heated gas remains transparent, and little IR radiation or organic aerosol production would be expected. As well as neatly explaining the 6 min delay of the main event, this hypothesis can also account for the hollow, crescent-shaped ejecta blankets observed by HST around the larger impact sites: material falling closer to the impact site does so at lower velocities, in general.

Given that the impacts occurred behind the planet's limb, and that the time for the impact site itself to rotate onto the limb ranged from $19 \mathrm{~min}$ for A to $9 \mathrm{~min}$ for $\mathrm{W}$ (see chapter by Chodas \& Yeomans), it must be asked how the re-entering plume material could be seen by earth-based observers only $6 \mathrm{~min}$ after impact. The answer lies in the fact that most of the debris was apparently ejected not vertically upward, as in the simple 'toy plume' model of Zahnle \& MacLow (1995), but in a south-easterly direction back along the inbound trajectory of the cometary fragments relative to the surface of Jupiter, and thus towards the limb. Although the simple ballistic model illustrated in Fig. 10 shows that material ejected at $45^{\circ}$ elevation and our nominal ejection velocity of $18 \mathrm{~km} \mathrm{~s}^{-1}$ takes $\sim 17 \mathrm{~min}$ to land, material ejected at lower velocities and on flatter trajectories lands sooner. Specifically, debris ejected at $18 \mathrm{~km} \mathrm{~s}^{-1}$ and $\theta=15^{\circ}$ re-enters after only $2 v_{e} \sin \theta / g=6 \mathrm{~min}$, but remains in direct view of the Earth, i.e., above the solid curves in Fig. 10. This is due in roughly equal parts to jovian rotation and downrange motion of the plume.

The overall duration of the typical main event of $\sim 10 \mathrm{~min}$ fits well with the period during which plume material ejected at $18 \mathrm{~km} \mathrm{~s}^{-1}$ and elevations ranging from $15^{\circ}$ to $45^{\circ}$ would have landed. Some material may have been ejected at steeper elevations, but the maximum observed plume height of $3200 \mathrm{~km}$ (Hammel et al. 1995) sets an upper limit of $\sim 12.5 \mathrm{~km} \mathrm{~s}^{-1}$ on the vertical velocity, and a corresponding upper limit of $2 v_{z} / g=17 \mathrm{~min}$ on the ballistic flight time.

The relation between time-of-flight and longitudinal distance travelled by the plume material is shown more clearly in Fig. 11. For simplicity, the azimuth of ejection is assumed to be a constant $45^{\circ}$ east of south, and the ejection velocity $v_{e}=17.9 \mathrm{~km} \mathrm{~s}^{-1}$. Points along the solid curve correspond to differing ejection angles $\theta$, starting with $\theta=0$ at the origin and increasing to $\theta=45^{\circ}$ at the maximum range of $12,800 \mathrm{~km}\left(10.5^{\circ}\right.$ in longitude on Jupiter at latitude $44^{\circ} \mathrm{S}$ ). (In reality, the full 3-dimensional plume involved a range of ejection velocities and azimuths as well, but these parameters are somewhat less important, within reasonable limits, in determining the maximum distance travelled downrange by the plume at any given time.) Horizontal dashed lines indicate significant events in the evolution of a typical impact plume: the start of the main event, the time at which the plume reaches maximum altitude, the time at which $\mathrm{CO}$ emission at $2.3 \mu \mathrm{m}$ appears in the spectrum (Meadows \& Crisp 1995), and the time of the shoulder in many lightcurves. Squares at $\theta=15^{\circ}$ and $\theta=30^{\circ}$ correspond approximately to the onset and peak of the main event, at which times the vertical velocity of the re-entering ejecta was 4.5 and $9 \mathrm{~km} \mathrm{~s}^{-1}$, respectively.

Superimposed on the time-of-flight curve are a set of straight diagonal lines showing the distances of several representative impact sites behind the limb as functions of time from impact. At the intersection of the appropriate diagonal line with the time-of-flight curve, the expanding curtain of re-entering ejecta crosses the limb onto the visible face of Jupiter. Ejecta from the $G$ impact, for example, began to land on the visible disk $6.3 \mathrm{~min}$ after impact, while that from the $\mathrm{R}$ impact crossed the limb within only $4.5 \mathrm{~min}$. 


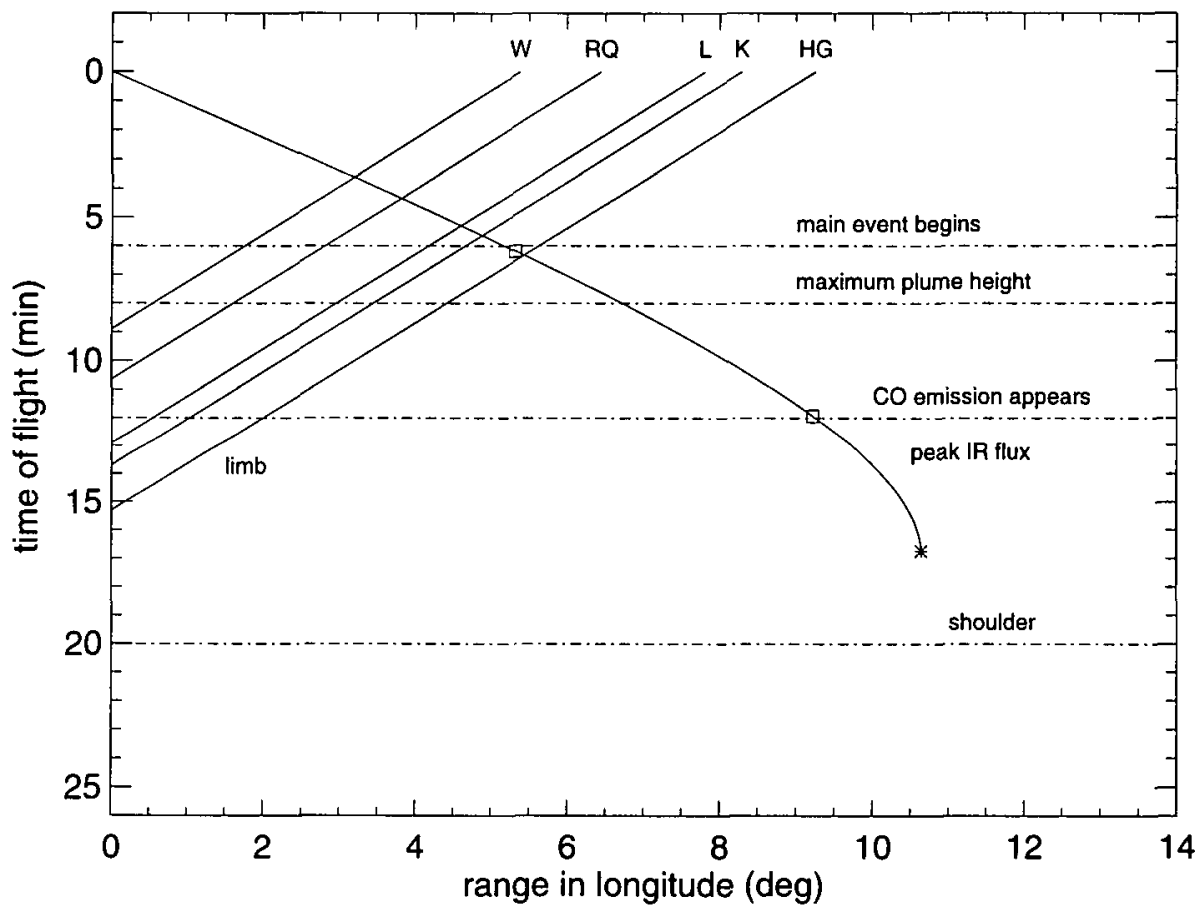

Figure 11. Time of flight vs. downrange distance (in longitude) for plume material ejected at $v_{e}=17.9 \mathrm{~km} \mathrm{~s}^{-1}$ and azimuth $45^{\circ}$ east of south, for a range of elevation angles $\theta$. An asterisk indicates the maximum range of $12,800 \mathrm{~km}$ (or $10.5^{\circ}$ in longitude) reached by ejecta at $\theta=45^{\circ}$, while the squares correspond to $\theta=15^{\circ}$ and $\theta=30^{\circ}$. Diagonal lines indicate where the falling ejecta crosses the limb onto the visible hemisphere of Jupiter.

The $\mathrm{G}$ and $\mathrm{R}$ impact sites themselves did not cross the limb until $15.5 \mathrm{~min}$ and $10.6 \mathrm{~min}$, respectively, as indicated by the intercept of the diagonal lines on the vertical axis.

\subsection{Shoulders}

The declining phases of many of the SL9 lightcurves show shoulders, in the form of either changes in slope or even secondary maxima, 19-20 min after impact (see examples in Figs. 1, 3 and 4). These shoulders are seen both at $2.3 \mu \mathrm{m}$ and at longer wavelengths. The $\mathrm{R}$ lightcurve also shows some evidence for subsequent, but more subdued shoulders at $\sim 28$ and 38 min after the impact; similar features are seen in the $\mathrm{C}$ and $\mathrm{G}$ lightcurves in Fig. 3.

The origin of these features is unclear. Suggestions that they are due to the impact ejecta crossing the terminator, and thus becoming visible in reflected light, run afoul of two facts: (i) the visibility of the shoulders at $12 \mu \mathrm{m}$ as well as the near-IR, and (ii) the observation that they appear at a more-or-less fixed time interval after impact. Most other explanations involve a dynamical response of the atmosphere to the re-entering debris, either in the form of a vertically propagating sound wave reflected from the tropopause and/or the upper stratosphere (Livengood et al. 1995; Nicholson et al. 1995a), or an actual re-launching of some of the ejecta (MacLow 1994, private communication). A 1-dimensional 'bounce' model, powered by the adiabatic compression of the atmosphere under the weight of the collapsing plume, was explored numerically by Deming et al. (1995) who obtained an oscillation period of $\sim 10 \mathrm{~min}$, in good agreement with the observations. Their model, however, ignored radiative damping and may thus have overestimated the amplitude of the bounce. Two-dimensional hydrodynamical 
models of the re-entering plume, including radiative losses, also show such a bounce (see chapter by MacLow in this volume).

\section{Temperatures}

Although a significant number of spectroscopic observations were made during several of the larger impacts, most of these data have yet to be published and few quantitative results are available at the time of writing. Compositional inferences from these measurements are reviewed in the chapter by Lellouch in this volume, while implications for the perturbed post-impact thermal structure of the jovian atmosphere are reviewed in the chapter by Conrath. We therefore restrict our discussion here to estimates of temperatures during the fireball and splashback phases, as these are of potential importance in constraining models of the impact process itself.

Temperature estimates may be divided into three categories, in order of decreasing probable reliability. First are physically-motivated spectroscopic estimates based, for example, on rotation-vibration line ratios. Even these estimates require a physical model for the emission region, and make assumptions of LTE, both of which may introduce significant errors (cf. discussion by Lellouch). Second are blackbody fits to regions of apparent continuum emission, perhaps representing the temperature of hot dust produced in the initial impact, or in the fallback shock. Figure 12 shows a series of mediumresolution grism spectra of the $\mathrm{K}$ impact obtained at the AAT which beautifully illustrate the evolution of the 2.0-2.4 $\mu \mathrm{m}$ spectrum during the fireball and splashback phases of this event (Meadows \& Crisp 1995). Third are simple color temperatures derived from photometric flux ratios, which may suffer large systematic errors if the measured flux in one or more of the bands used is in fact dominated by line emission. We will group the available estimates by impact phase.

\subsection{First precursor (the bolide?)}

Very few multi-wavelength observations, and little spectroscopic data, have been reported for this short-lived phase in the lightcurves. Peak fluxes for the $\mathrm{R}$ impact first precursor at 2.3, 3.2 and $4.5 \mu \mathrm{m}$ (cf. Fig. 1(b)) are consistent with a color temperature of $1000 \pm 120 \mathrm{~K}$ (Nicholson et al. 1995b). A comparison of the flux measured by HST at $0.89 \mu \mathrm{m}$ for what is interpreted as the meteor phase of the G impact (Hammel et, al. 1995) with the simultaneously-measured first precursor brightness at $2.3 \mu \mathrm{m}$ (cf. Fig. 7) yields a color temperature of $1800 \mathrm{~K}$ (McGregor et al. 1995). However, in both cases the measurements themselves were not made simultaneously, and it is likely that the fluxes changed appreciably during the integration times of 5-30 sec, casting considerable doubt on the reliability of the derived temperatures. The $\mathrm{K}$ impact spectrum at this time shows elevated emission longward of $2.05 \mu \mathrm{m}$, which matches a $6700 \mathrm{~K}$ blackbody (Fig. 12, panel 3).

\subsection{Second precursor (rising fireball)}

Thermal emission from the rising fireball was detectable for several minutes in the case of the larger impacts, permitting some spectroscopic observations. During most of the second precursor phase (Fig. 12, panels 4-6), the $\mathrm{K}$ impact spectra show primarily continuum emission with a blackbody temperature which declined from $720 \mathrm{~K}$ at $T_{i}+3.5 \mathrm{~min}$ to $420 \mathrm{~K}$ at $T_{i}+6 \mathrm{~min}$. This presumably reflects the temperature of the plume after it had risen into view from the Earth; Galileo NIMS observations for the G and R impacts show considerably higher temperatures in the first $30 \mathrm{sec}$ of the plume's development, when it was still hidden behind the limb (Carlson et. al. 1995a). 
K: 10:22:06 U.T

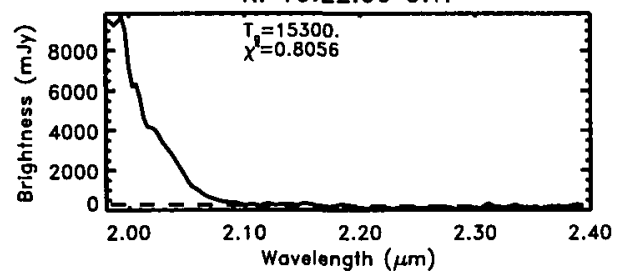

K: 10:22:42 U.T.

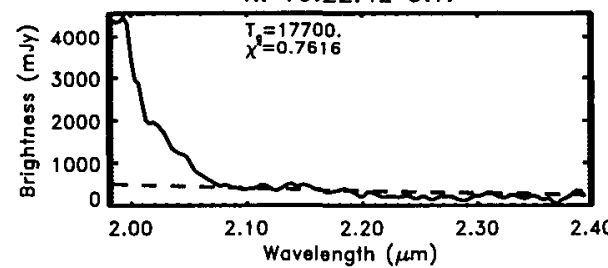

K: 10:23:57 U.T

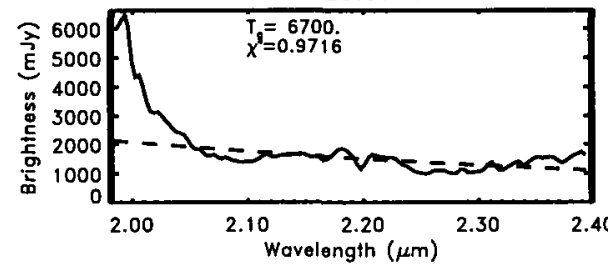

K: 10:25:10 U.T.

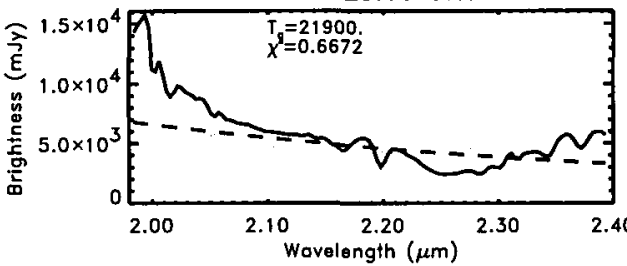

K: 10:27:32 U.T.

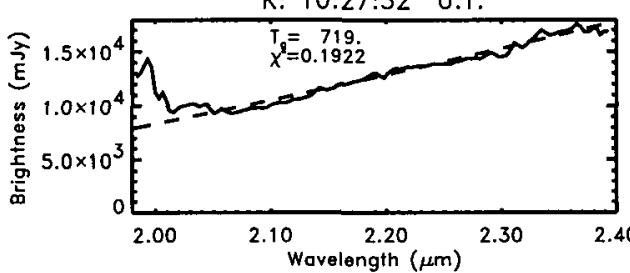

K: 10:30:34 U.T.

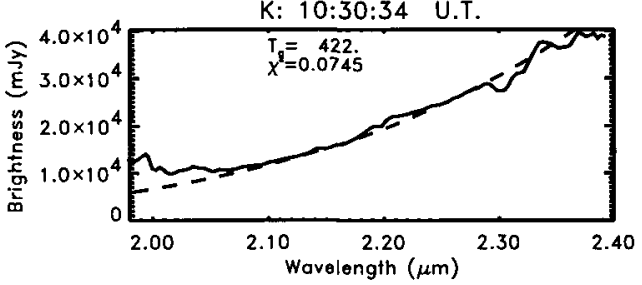

K: 10:31:31 U.T.

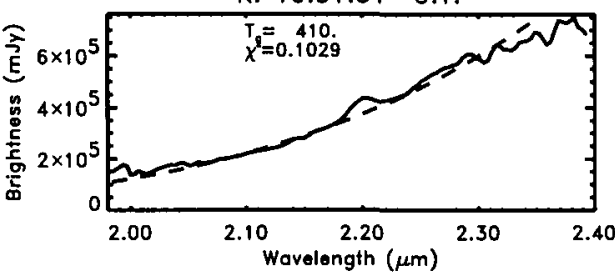

K: $10: 32: 20 \quad$ U.T.

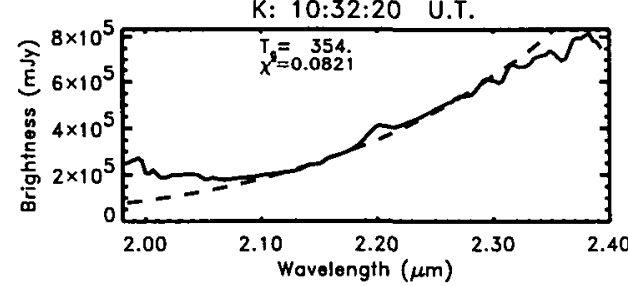

K: $10: 36: 13$ U.T

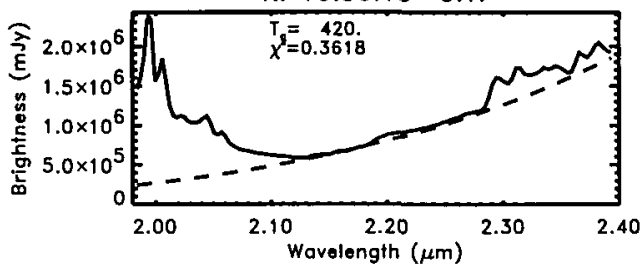

K: 10:38:57 U.T.

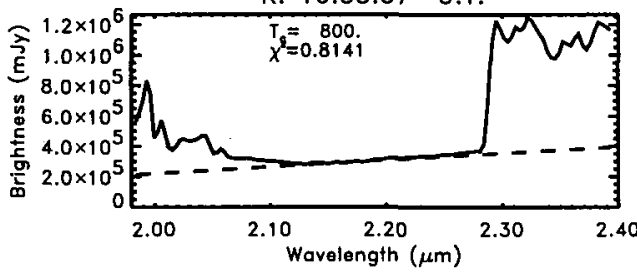

K: 10:40:33 U.T.

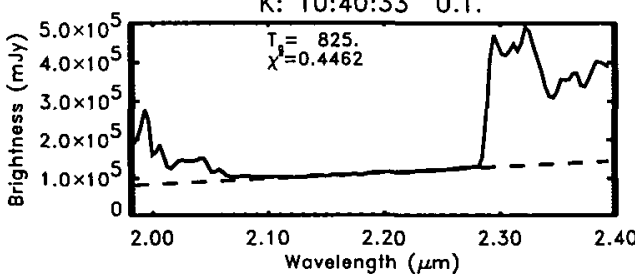

K: 10:53:47 U.T.

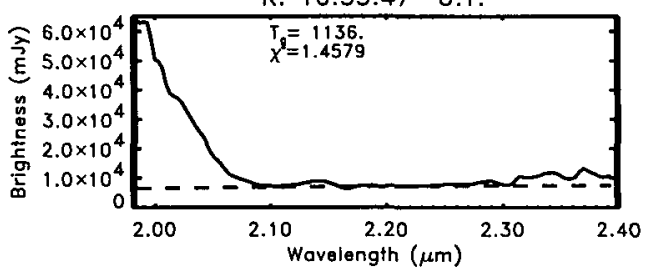

Figure 12. 2.0-2.4 $\mu \mathrm{m}$ spectra obtained at the Anglo-Australian Telescope during the $\mathrm{K}$ impact. Dashed curves indicate blackbody fits to the continuum regions of the spectra. The Galileo impact time was $T_{i}=10: 24: 13 \mathrm{UT}$, while the main event began at $\sim 10: 31$. Pre-impact leader emission is seen in panels $1 \& 2$, and the first precursor in panel 3. The second precursor begins in panel 4 and continues through panels 5 and 6 . Panels $7-11$ cover the main event, with panel 9 at $T_{i}+12 \mathrm{~min}$ falling near the peak of the $2.3 \mu \mathrm{m}$ lightcurve. Absolute calibration is provisional. Adapted from Meadows \& Crisp (1995). 
Color temperatures derived from 2.3/3.1 $\mu \mathrm{m}$ flux ratios during the latter part of the $\mathrm{H}$ second precursor are 900-1300 K (Hamilton et al. 1995), while during the equivalent phase of the $\mathrm{R}$ impact the $2.3 / 4.5 \mathrm{um}$ color temperature slowly increased from $650 \mathrm{~K}$ at $T_{i}+1 \mathrm{~min}$ to $850 \mathrm{~K}$ at $T_{i}+3.5 \mathrm{~min}$. Spanning a wider wavelength range, measurements of the $H$ and $\mathrm{L}$ precursor peaks at 10 and $12 \mu \mathrm{m}$ (Livengood et al. 1995; Lagage et al. 1995) combined with data at at $2.3 \mu \mathrm{m}$ imply similar color temperatures of $600-625 \mathrm{~K}$.

\subsection{Main event, (collapsing plume)}

In the early stages of the main event, the 2.0-2.4 $\mu \mathrm{m}$ spectrum of the $\mathrm{K}$ impact continued to be dominated by continuum emission, with temperatures of $\sim 400 \mathrm{~K}$. Beginning near the time of peak flux, and for $\sim 10 \mathrm{~min}$ thereafter, prominent $\mathrm{CO}$ emission was observed longward of $2.30 \mu \mathrm{m}$, with $\mathrm{NH}_{3}$ and possible $\mathrm{H}_{2} \mathrm{O}$ emission below $2.1 \mu \mathrm{m}$ (Meadows \& Crisp 1995). Similar CO emission was observed in near-IR spectra of the C, D, G, $\mathrm{H}, \mathrm{R}$ and $\mathrm{W}$ impacts (Meadows \& Crisp 1995; Herbst et al. 1995). Excitation of these $\Delta \nu=2$ vibrational bands usually implies a temperature of $2000 \mathrm{~K}$ or higher. The $\mathrm{NH}_{3}$ emission implies a similarly high temperature, although Lellouch cautions against such a simple LTE-based interpretation. In contrast, fits to the emission by $\mathrm{H}_{2} \mathrm{O}$ at $2.04 \mu \mathrm{m}$ in the spectra at the $\mathrm{K}$ peak suggest a temperature of $600-700 \mathrm{~K}$ (Meadows \& Crisp 1995), while Encrenaz et al. (1995) estimate a temperature of $750 \mathrm{~K}$ at the peak of the $\mathrm{H}$ lightcurve, based on $\mathrm{CH}_{4}$ spectra at $3.5 \mu \mathrm{m}$ and an assumed effective pressure level for the radiating region of $1 \mathrm{mb}$. Maillard et al. (1995) derived a temperature of 750-1500 K at the peak of the $\mathrm{C}$ lightcurve from $\mathrm{CH}_{4}$ emission lines at $3.3 \mu \mathrm{m}$. The onset of $\mathrm{CO}$ emission may signal the increasing vertical re-entry velocity of the plume, $v_{z}=g t / 2$ : by $T_{i}+12 \mathrm{~min}$ this had reached $9 \mathrm{~km} \mathrm{~s}^{-1}$.

Bjoraker et al. (1995), using the Kuiper Airborne Observatory with an echelle grating spectrometer at $7.7 \mu \mathrm{m}$, observed the main events for the $\mathrm{G}$ and $\mathrm{K}$ impacts. Emission due to hot methane, water and dust was observed to commence $\sim 7 \mathrm{~min}$ after the $\mathrm{K}$ impact, peak at 13-14 min post-impact, and then decay by $\sim 25$ min. A temperature of $\sim 1000 \mathrm{~K}$ and a base pressure of $3 \mu \mathrm{b}$ at the $\mathrm{K}$ peak was derived from the relative strengths of hot $\mathrm{H}_{2} \mathrm{O}$ and $\mathrm{CH}_{4}$ lines. Lower-resolution 5-10 $\mu \mathrm{m}$ spectroscopic observations were also made with the KAO for the $\mathrm{R}$ and $\mathrm{W}$ impacts by Sprague $e$ t al. (1995), revealing strong emission by $\mathrm{CH}_{4}$ and $\mathrm{H}_{2} \mathrm{O}$ at a temperature of $\sim 500 \mathrm{~K}$.

Simultaneous monitoring of the $\mathrm{R}$ lightcurve at 3.2 and $4.5 \mu \mathrm{m}$ showed a surprisingly constant color temperature of $\sim 1000 \mathrm{~K}$ during the fallback phase, although this may be strongly affected by $\mathrm{CH}_{4}$ line emission at $3.2 \mu \mathrm{m}$. In fact, the best overall fit to the $\mathrm{R}$ impact spectrum from 2.3 to $13 \mu \mathrm{m}$ at the time of peak flux implies either a blackbody at $700 \mathrm{~K}$, or an opacity which scales as $\lambda^{-1}$, as expected for emission by sub-micron dust particles, and $T \sim 600 \mathrm{~K}$ (Nicholson et al. 1995b). Simultaneous narrowband measurements at $7.85,10.3$ and $12.2 \mu \mathrm{m}$ by Friedson et al. (1995) are also consistent with this fit (cf. Fig. 13).

Color temperatures derived from published lightcurves at 2.3 and $10-12 \mu \mathrm{m}$ for the $\mathrm{E}$, $\mathrm{H}, \mathrm{L}$ and $\mathrm{R}$ impacts, evaluated at the time of peak flux, also fall in the range $550-700 \mathrm{~K}$. Of course, these estimates may be systematically skewed by $\mathrm{CO}$ emission at $2.3 \mu \mathrm{m}$ and/or silicate emission at $10 \mu \mathrm{m}$ (Nicholson et al. 1995b). Perhaps none of the above blackbody temperature estimates should be taken too seriously until a physical model of the emission from the shocked mixture of cometary debris and jovian atmosphere is developed which takes into account both molecular and dust emission, as well as the near-limb viewing geometry. 


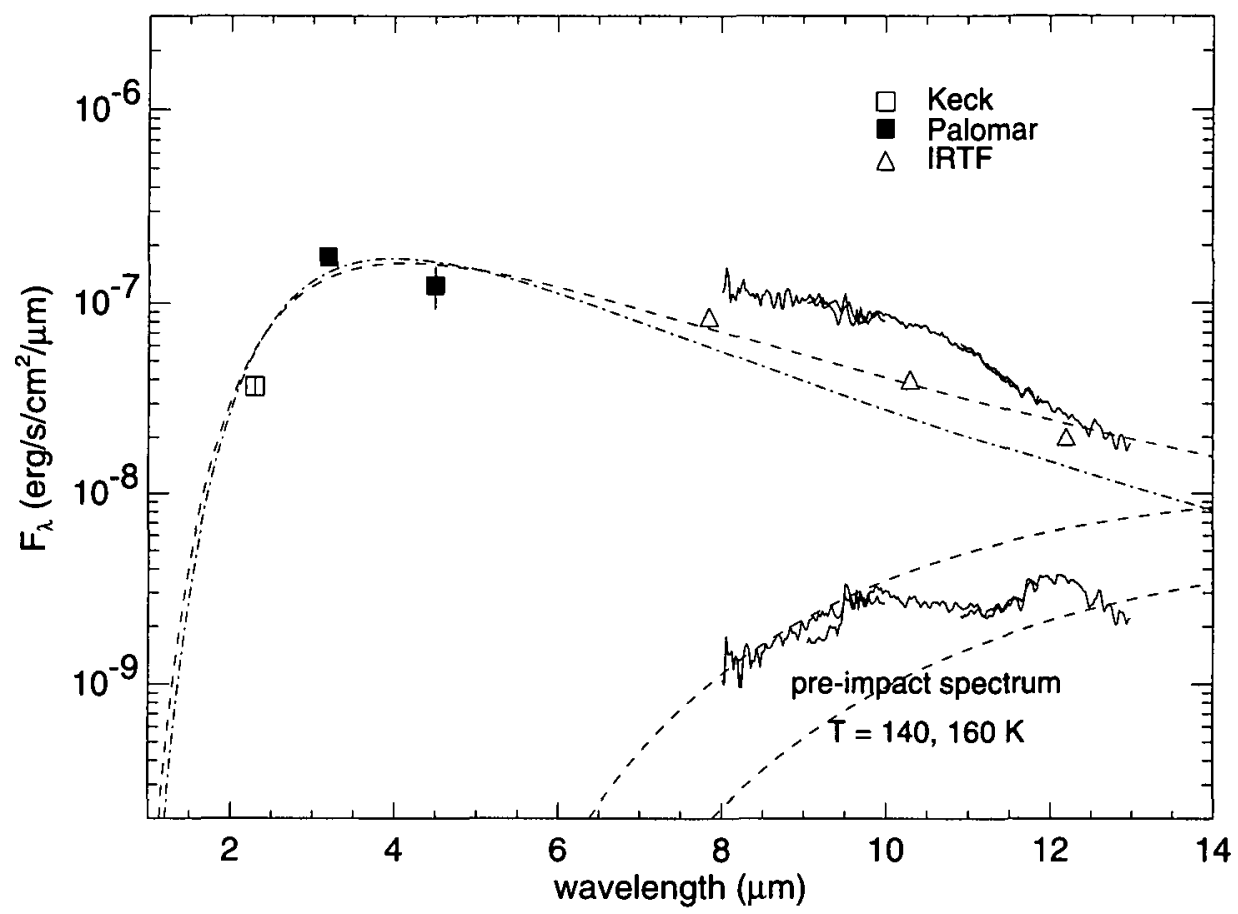

Figure 13. Composite spectrum for the $\mathrm{R}$ impact, at the peak of the main event, based on narrowband imaging at $2.3,3.2,7.85,10.3$ and $12.2 \mu \mathrm{m}$, broadband imaging at $4.5 \mu \mathrm{m}$, and low-resolution spectroscopy at 8-13 $\mu \mathrm{m}$. Data from Nicholson et al. (1995a), Graham et al. (1995) and Friedson et al. (1995). The dashed curve shows the best-fitting blackbody spectrum $(700 \mathrm{~K}$, with an optical depth-solid angle product $\Omega \tau=0.01 \mathrm{sq}$ arcsec), while the dot-dashed curve is the best-fitting dust-opacity $\left(\tau \sim \lambda^{-1}\right)$ model, with $T=600 \mathrm{~K}$ and $\Omega \tau=0.01 \mathrm{sq}$ arcsec at $10 \mu \mathrm{m}$. The spectrum at lower right was obtained immediately prior to the impact, and reflects normal jovian tropospheric and lower stratospheric temperatures of 140-160 K. Adapted from Nicholson et al. (1995b).

\section{Anomalous lightcurves}

A few apparently aberrant lightcurves do not appear to fit comfortably into the scenario described in $\S 3$. The strong Q1 precursor, similar in shape to the second L precursor, is reported to have started $37 \mathrm{sec}$ before the inital Galileo PPR detection (Herbst et al. 1995), an anomaly with no obvious explanation.

The Q2 impact, which occurred 29 min before Q1, showed a single precursor followed about $8 \mathrm{~min}$ later by a very subdued 'main event', which was fainter than the precursor (Herbst et al. 1995).

The $\mathrm{V}$ impact was observed only as a brief flash, $\sim 30 \mathrm{sec}$ in duration, with no subsequent main event at all (Weinberger 1994; Meadows 1994, private communications). Based on its duration and its similarity to the first $\mathrm{R}$ precursor at $2.3 \mu \mathrm{m}$ (Fig. 14), we tentatively identify this as a 'first precursor', perhaps due to the entry flash of a particularly small and/or weak fragment of SL9.

For each of the $B, M$ and $N$ impacts, there is a single report of a brief $(1-2 \mathrm{~min}$ in duration) period of weak $2.3 \mu \mathrm{m}$ emission (de Pater 1994, private comunication; McGregor et al. 1995), which most likely reflects a very faint main event with no discernable precursor. The $\mathrm{N}$ event was first seen at 10:36:30 UT, $7 \mathrm{~min}$ after the impact flash was detected by the Galileo SSI instrument, consistent with a barely-detectable main event. 


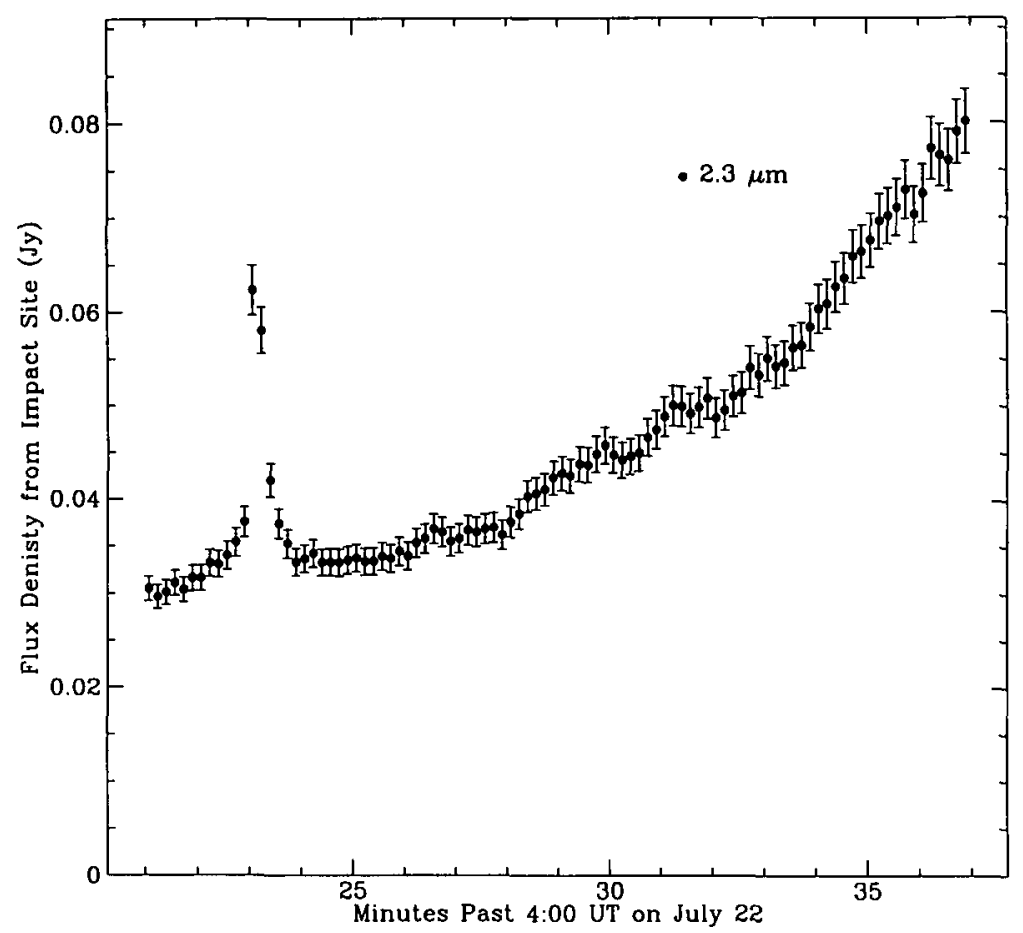

FIgURE 14. Palomar lightcurve showing the very brief $\mathrm{V}$ impact flash at $2.3 \mu \mathrm{m}$, at $10 \mathrm{sec}$ resolution. The increasing background signal is due to the old $\mathrm{E}$ impact site rotating across the terminator. A simultaneous flash was recorded at the AAT. Possible faint leader emission is seen before the main flash, but there appears to have been no detectable 'main event'. Unpublished data from A. Weinberger.

A similar report of a faint $U$ event was made by Cochran et al. (1995), but simultaneous observations with larger telescopes under clear skies failed to detect any signature of this impact (Hamilton et al. 1995). Likewise, early reports of an F impact have not been confirmed by other observers, and were likely due to confusion with the re-appearance of the old $\mathrm{E}$ impact site on the limb.

\section{Other phenomena}

\subsection{Satellite flashes}

Considerable attention before and during the SL9 impacts was devoted to predicting and searching for impact flashes reflected from the Galilean satellites. Despite several efforts to record a reflected flash with photometers and CCD cameras, no unambiguous reflected signals were detected. Consolmagno \& Menard (1995) monitored Europa during the A impact and Europa during the H, Q1 and Q2 impacts. Possible 2- $\sigma$ detections of flashes from A, Q2 and Q1 were reported, but none was considered to be convincing. A'Hearn et al. (1995) and Woodney et al. (1995) report observations of Io and Europa during the $\mathrm{D}, \mathrm{E}$ and $\mathrm{K}$ impacts, and derive upper limits on the peak flash luminosity at 0.42 and $0.62 \mu \mathrm{m}$. However, the $\mathrm{D}$ and $\mathrm{E}$ limits far exceed the peak luminosities measured by the Galileo PPR and SSI instruments for several brighter impacts. It is apparent from these results that the flashes-particularly when reflected from sunlit satellites-were far too faint for detection at visual wavelengths.

A more favourable opportunity was provided by the $\mathrm{K}$ impact, which occurred while Europa was in eclipse but visible to earth-based observers if illuminated by the flash. 
However, no detectable visual signal was observed, and the flux at $2.34 \mu \mathrm{m}$ did not exceed $6 \mathrm{mJy}$ (McGregor et al. 1995). Both visible and near-IR upper limits on the $\mathrm{K}$ flash exceed by a factor of $\sim 10$ those expected based on the peak luminosity measured by the Galileo SSI experiment, assuming isotropic emission and temperatures of several thousand $\mathrm{K}$.

\subsection{Post-impact features on Jupiter}

Due to the strong methane absorption in the underlying atmosphere, the ejecta blankets deposited by the larger impacts were readily visible in reflected sunlight as bright features in images taken at wavelengths of 2.3 or $3.5 \mu \mathrm{m}$. The structure of the ejecta blankets is best seen in the HST images (cf. chapter by Hammel in this volume), but some information concerning the impact process itself may be gleaned from the near-IR images. Although the impact site was generally unresolved in images taken near the peak of the main event, implying a source size of order $4000 \mathrm{~km}$ or less, later images frequently showed an extended region of emission, elongated along the planet's limb (Graham et al. 1995; Nicholson et al. 1995a; Lagage et al. 1995). Dimensions ranged from $\sim 8000 \mathrm{~km}$ for the $\mathrm{R}$ impact at 2.3 and $4.5 \mu \mathrm{m}$ to $30,000 \mathrm{~km}$ for $\mathrm{L}$ at $12 \mu \mathrm{m}$. The smaller measurement is compatible with the size of the ejecta blankets imaged in the visible by HST (Hammel et al. 1995).

Near-IR images of several sites taken during the impact week show a distinct central spot and a crescent-shaped feature to the south-east, reminiscent of the celebrated HST image of the $\mathrm{G}$ site (West et al. 1995). Although only a few quantitative analyses have yet been performed using these data (Banfield et al. 1995; Chanover et al. 1995; Ortiz et al. 1995; Moreno et al. 1995), it is clear that the ejecta was deposited fairly high in the stratosphere, at pressure levels of $10 \mathrm{mb}$ or less. For more details, see the chapter by West in this volume.

\subsection{Three-micron rings}

More unexpected was the discovery of very large circular rings surrounding the $C, G$ and $\mathrm{K}$ impact sites and visible only between 3.1 and $4.0 \mu \mathrm{m}$ (McGregor et al. 1995). Over this range, their spectra are extremely blue. Measurements of re-projected images show that the $G$ and $K$ rings expanded at a rate of $\sim 1400 \mathrm{~m} \mathrm{~s}^{-1}$ to radii of $\sim 18,500 \mathrm{~km} 2 \mathrm{hr}$ after the impacts, before slowly fading from view 1.5 hrs later. No sign of these features is seen in many images taken at shorter wavelengths. The rings are centered $\sim 3500 \mathrm{~km}$ to the south-east of the impact sites, in the same direction as the displacement of the HST ejecta patterns, and appear to have expanded from an initial radius of $\sim 8000 \mathrm{~km}$, within the optical crescent. It is not clear if the rings are seen in emission or reflected sunlight, although the former seems more consistent with the rapid fading. Their spectral signature is consistent with emission by poly-HCN (P. Wilson 1995, private communication). These rings are quite distinct from the expanding waves seen in the HST images of several sites; the latter are restricted to radii of $5000 \mathrm{~km}$ or less and expanded at a much slower rate of $450 \mathrm{~m} \mathrm{~s}^{-1}$ (Hammel et al. 1995).

Although no good model exists for these features, it has been suggested (Zahnle 1995, private communication) that they could be due to impact debris 'sliding' horizontally across the top of the atmosphere, from an initial location in the crescent. Alternative explanations include mildy supersonic shock waves propagating in the stratosphere, where the sound speed is $\sim 980 \mathrm{~m} \mathrm{~s}^{-1}$. 


\section{Summary}

Despite some predictions to the contrary, almost all phases of the impact process--with the exception of the terminal phase of the bolide and the initial seconds of the fireballwere apparently recorded by ground-based instruments. Although we are still far from having quantitative models which successfully account for all the features observed in the SL9 impact lightcurves, a viable qualitative picture of the various impact phenomena does seem to have emerged, capable of explaining the principal observations. A remarkably consistent set of characteristics emerges for the best-observed events, beginning with the first and second precursor flashes as the bolide entered the jovian atmosphere and the hot fireball emerged from behind the limb, and culminating in the infrared main event as the ejecta plume re-entered the jovian atmosphere. Only a handful of weaker events failed to follow this pattern. A simple ballistic plume model is successful in accounting for the timing of the major features in the lightcurves; in the future more realistic 3-D hydrodynamic simulations will be required to model quantitatively the observed fluxes and extract fragment energies, penetration depths, re-entry altitudes, etc. Particular problems include the persistence of the second precursor for the larger impacts, and the multiple shoulders exhibited by several lightcurves.

A general correlation is observed between the peak brightness of the infrared signal and the estimated sizes of the pre-impact fragments, and with the morphology of the postimpact sites as seen in the HST images. The faintest (or undetected) impact signatures were associated with fragments displaced from the main SL9 train, strongly suggesting that some 'fragments' were little more than fluffy aggregates, or perhaps simply agglomerations of dust.

Temperature estimates derived from near-IR and mid-IR photometry and spectroscopy range from $1000 \mathrm{~K}$ or higher for the entry trail of the bolide, through $\sim 750 \mathrm{~K}$ for the fireball after it had cleared the limb. Temperatures derived during the splashback phase are less concordant, varying from $500 \mathrm{~K}$ to $2000 \mathrm{~K}$. This dispersion may reflect a combination of non-LTE effects, non-blackbody emission, and real temperature variations, both spatial and temporal. Development of more realistic radiative models for the reentering plume is clearly a high priority if the wealth of available spectroscopic and color data is to be fully interpreted.

Over the year since the events of July 1994 the author has greatly benefitted from discussions with many individuals, and it is impossible at this date to attribute correctly all original ideas. Much of the impact scenario presented here was developed in discussions at the AAS Division for Planetary Sciences conference in October 1994 and the European SL9 meeting in February 1995, and refined during IAU Colloquium 156 in May 1995. Particular thanks go to Don Banfield, Clark Chapman, Paul Chodas, Imke de Pater, Doug Hamilton, Tom Hayward, Peter McGregor, Keith Matthews, Vikki Meadows, Gerry Neugebauer, Glenn Orton, Alycia Weinberger, Kevin Zahnle and, last but not least, Audrey and Alicia. Preparation of this article was supported by the NSF and the NASA Planetary Astronomy program.

\section{REFERENCES}

A'Hearn, M. F., Meier, R., Wellnitz, D., And Woodney, L. 1995 Were Any Impact Flashes Seen in Reflection from the Satellites? In Proceedings of the European SL-9/Jupiter Workshop (eds. R. West and H. Bohnhardt) pp. 113-118. ESO. 
Banfield, D., Gierasch, P., Squyres, S. W., Nicholson, P. D., Conrath, B. AND MatThews, K. $19952 \mu \mathrm{m}$ Spectrophotometry of Jovian Stratospheric AerosolsScattering Opacities, Vertical Distributions and Wind Speeds. Icarus, (submitted).

Bjoraker, G. L., Stolovy, S. R., Herter, T. L., Gull, G. E. ANd Pirger, B. E. 1995 Detection of Water After the Collision of Fragments G and K of Comet Shoemaker-Levy 9 with Jupiter. Science (submitted).

Boslough, M., Crawford, D., Robinson, A., and Trucano, T. 1994 Mass and penetration depth of Shoemaker-Levy 9 fragments from time-resolved photometry. Geophys. Res. Lett. 21, 1555.

Boslough, M., Crawford, D., Trucano, T., and Robinson, A. 1995 Numerical modeling of Shoemaker-Levy 9 impacts as a framework for interpreting observations. Geophys. Res. Lett. 22, 1821.

Carlson, R. W., Weissman, P. R., Segura, M., hui, J., Smythe, W. D., Johnson, T., Baines, K. H., Drossart, P., Encrenaz, Th., And Leader, F. E. 1995a Galileo infrared observations of the Shoemaker-Levy $9 \mathrm{G}$ impact fireball: A preliminary report. Geophys. Res. Lett. 22, 1557.

Carlson, R. W., Weissman, P. R., Hui, J., Smythe, W. D., Baines, K. H., Johnson, T. V., Drossart, P., Encrenaz, T., Leader, F. E. and Mehlman, R. 1995b Some timing and spectral aspects of the $\mathrm{G}$ and $\mathrm{R}$ collision events as observed by the Galileo nearinfrared mapping spectrometer. In Proceedings of the European SL-9/Jupiter Workshop (eds. R. West and H. Bohnhardt) pp. 69-73. ESO.

Chanover, N. J., Beebe, R. F., Murrell, A. S., And Simon, A. A. 1995 Absolute Reflectivity Spectra of Jupiter: 0.25-3.5 Microns. Icarus, (submitted).

Chapman, C. R., Merline, W. J., Klaasen, K., Johnson, T. V., Heffernan, C., Belton, M. J. S., Ingersoll, A. P., And the Galileo Imaging Team 1995 Preliminary results of Galileo direct imaging of S-L 9 Impacts. Geophys. Res. Lett. 22, 1561.

Cochran, A. L., Armosky, B. J., Pulliam, C. E., Clark, B. E., Cochran, W. E., Frueh, M., Lester, D. F., Trafton, L., Kim, Y., NA, C., AND Pryor, W. 1995 An Update on Imaging Observations from McDonald Observatory. In IAU Colloquium 156: The Collision of Comet P/Shoemaker-Levy 9 and Jupiter, p. 21.

Colas, F., Tiphene, D., Lecacheux, J., Drossart, P., deBatz, D., Pau, S., Rouan, D., AND SeVRe, F. 1995 Near-infrared imaging of SL9 impacts on Jupiter from Pic-du-Midi Observatory. Geophys. Res. Lett. 22, 1765.

Consolmagno, G. J. And Menard, G. 1995 A search for variations in the light curves of Io and Europa during the impact of Comet SL9: A, H, and Q events. Geophys. Res. Lett. 22, 1633.

Crawford, D., Boslough, M., Trucano, T., and Robinson, A. 1994. The impact of comet Shoemaker-Levy 9 on Jupiter. Shock Waves, 4, 47.

Deming, D And Harrington, J. 1995. no title; poster paper at IAU Colloquium 156, Baltimore MD.

Drossart, P., Encrenaz, T., Lecacheux, J., Colas, F., and Lagage, P.O. 1995 The time sequence of SL9/impacts H and L from infrared observations. Geophys. Res. Lett. 22, 1769.

Encrenaz, T., Schulz, R., Stüwe, J. A., Wiedemann, G., Drossart, P., and Crovisier, J. 1995 Near-IR spectroscopy of Jupiter at the time of SL9 impact: Emissions of $\mathrm{CH}_{4}, \mathrm{H}_{3}^{+}$, and $\mathrm{H}_{2}$. Geophys. Res. Lett. 22, 1577.

Friedson, A. J., Hoffmann, W. F., Goguen, J. D., Deutsch, L. K., Orton, G. S., Hora, J. L., Dayal, A., Spitale, J. N., Wells, W. K., and Fazio, G. G. 1995 Thermal infrared lightcurves of the impact of comet Shoemaker-Levy 9 Fragment R. Geophys. Res. Lett. 22, 1569.

Graham, J., de Pater, I., Jernigan, J., LiU, M., and Brown, M. 1995. W. M. Keck telescope observations of the comet $\mathrm{P} /$ Shoemaker-Levy 9 fragment $\mathrm{R}$ Jupiter collision. Science 267, 1320. 
Hamilton, D. P., Herbst, T. M., Richichi, A., Böhnhardt, H., Ortiz, J. L. 1995 Calar Alto Observations of Shoemaker-Levy 9: Characteristics of the H and L Impacts. Geophys. Res. Lett. 22, 2417.

Hammel, H. B., Beebe, R. F., Ingersoll, A. P., Orton, G. S., Mills, J. R., Simon, A. A., Chodas, P., Clarke, J. T., De Jong, E., Dowling, T. E., et al. 1995 HST Imaging of Atmospheric Phenomena Created by the Impact of Comet Shoemaker-Levy 9. Science 267, 1288.

Hasegawa, H., Takeuchi, S., Watanabe, J. 1995 Grain Formation in Cometary Impact Plume. In Proceedings of the European SL-9/Jupiter Workshop (eds. R. West and H. Bohnhardt) pp. 279-286. ESO.

Herbst, T. M., Hamilton, D. P., Böhnhardt, H., and Ortiz-Moreno, J. L. 1995 Near Infrared Imaging and Spectroscopy of the SL-9 Impacts from Calar Alto. Geophys. Res. Lett. 22, 2413.

Hord, C. W., Pryor., W. R., Stewert, A. I. F., Simmons, K. E., Gebben, J. J., Barth, C. A., McClintock, W. E., Esposito, L. W., Tobiska, W. K., West, R. A., EdBERg, S. J., AJello, J. M. AND NaviauX, K. L. 1995 Direct observations of the comet Shoemaker-Levy 9 fragment G impact by Galileo UVS. Geophys. Res. Lett. 22, 1565.

Lagage, P. O., Galdemard, Ph., Pantin, E., Jouan, R., Masse, P., Sauvage, M., Olofsson, G., Huldtgren. M., Nordh, L., Belmonte, J. A., Regulo, C., Rodriguez Espinosa, J. M., Vidal, L., Mosser, B., Ulla, A., And Gautier, D. 1995 SL-9 fragments A, E, H, L, Q1 collision on to Jupiter: Mid-infrared light curves. Geophys. Res. Lett. 22, 1773.

Livengood, T. A., Käufl, H. U., Kostiuk, T., Bjoraker, G. L., Romani, P. N., WiedeMann, G., Mosser, B., AND Sauvage, M. 1995 Multi-Wavelength Thermal-Infrared Imaging of SL9 Impact Phenomena. In Proceedings of the European SL-9/Jupiter Workshop (eds. R. West and H. Bohnhardt) pp. 1437-146. ESO.

Maillard, J. P., Drossart, P., Bézard, B., de Bergh, C., Lellouch, E., Marten, A., Caldwell, J., Hilico, J. C. 1995 Methane and carbon monoxide infrared emissions observed at the CFH telescope during the collision of comet SL-9 on Jupiter. Geophys. Res. Lett 22, 1573.

Martin, T. Z., Orton, G. S., Travis, L. D., Tamppari, L. K., and Claypool, I. 1995 Observation of Shoemaker-Levy Impacts by the Galileo Photopolarimeter radiometer. Science 268, 1875.

MCGregor, P., Nicholson, P. D., AND Allen, M. 1995. CASPIR observations of the collision of Comet Shoemaker-Levy 9 with Jupiter. Icarus, (submitted).

Meadows, V. AND CRISP, D. 1995 Impact Plume Composition from Near-Infrared Spectroscopy. In Proceedings of the European SL-9/Jupiter Workshop (eds. R. West and H. Bohnhardt) p. 239. ESO.

Meadows. V., Crisp, D., Orton, G., Brooke, T., And Spencer, J. 1995 AAT IRIS Observations of the SL-9 Impacts and Initial Fireball Evolution. In Proceedings of the European SL-9/Jupiter Workshop (eds. R. West and H. Bohnhardt) p. 233. ESO.

Moreno, F., Munoz, O., Molina, A., Lopez-Moreno, J. J., Ortiz, J. L., Rodriguez, J., Lopez-Jiminez, A., Girela, F., Larson, S. M. And Campins, H. 1995 Physical properties of the aerosol debris generated by the impact of fragment $\mathrm{H}$ of comet $\mathrm{P} /$ ShoemakerLevy 9 on Jupiter. Geophys. Res. Lett. 22, 1609.

Nicholson, P. D., Gierasch, P. J., Hayward, T. L., McGhee, C. A., Moersch, J. E., Squyres, S. W., Van Cleve, J., Matthews, K., Neugebauer, G., Shupe, D., WeinBerger, A., Miles, J. W., AND Conrath, B. J. 1995a Palomar observations of the impact of fragment $\mathrm{R}$ of comet P/Shoemaker-Levy 9: I. Light curves. Geophys. Res. Lett. 22, 1613.

Nicholson, P. D., Gierasch, P. J., Hayward, T. L., McGhee, C. A., Moersch, J. E., Squyres, S. W., Van Cleve, J., MatThews, K., Neugebauer, G., Shupe, D., WeinBerger, A., Miles, J. W. AND ConRath, B. J. 1995b Palomar observations of the impact of fragment R of comet P/Shoemaker-Levy 9: II. Spectra. Geophys. Res. Lett. 22, 1617. 
Ortiz, J. L., Munoz, O., Moreno, F., Molina, A., Herbst, T. M., Birkle, K., BöhnhardT, H., AND Hamilton, D. P. 1995 Models of the Shoemaker-Levy 9 Collision Generated Hazes. Geophys. Res. Lett 22, 1605.

Orton, G., A'Hearn, M., Baines, K., Deming, D., Dowling, T., Goguen, J., GrifFith, C, Hammel, H., Hoffmann, W., Hunten, D., Jewitt, D., Kostiuk, T., et al. 1995 Collision of Comet Shoemaker-Levy 9 with Jupiter Observed by the NASA Infrared Telescope Facility. Science 267, 1277.

Sekanina, Z., Chodas, P. W., And Yeomans, D. K. 1994 Tidal Disruption and the Appearance of Periodic Comet Shoemaker-Levy 9. Astron. \& Astrophysics 289, 607.

Skrutskie, M. F., AND AAs, S. 1995 A $2.3 \mu \mathrm{m}$ Light Curve of the "L" impact. Icarus (submitted).

Sprague, A., Bjoraker, G., Hunten, D., Witteborn, F., Kozlowski, R., and Wooden, D. 1995. Observations of $\mathrm{H}_{2} \mathrm{O}$ following the $\mathrm{R}$ and $\mathrm{W}$ impacts of comet Shoemaker-Levy 9 into Jupiter's atmosphere. Icarus (in press).

Takata, T., O'Keefe, J. D., Ahrens, T. J., And Orton, G. 1994 Comet Shoemaker-Levy 9: Impact on Jupiter and plume evolution. Icarus 109, 3.

Takeuchi, S., Hasegawa, H., Watanabe, J., Yamashita, T., Abe, M., Hirota, Y., Nishihara, E., OKumUra, S., AND MoRI, A. 1995 Near-IR imaging observations of the cometary impact into Jupiter: Time variation of radiation from impacts of fragments $\mathrm{C}$, D, and K. Geophys. Res. Lett. 22, 1581.

Watanabe, J., Yamashita, T., Hasegawa, H., Takeuchi, S., Abe, M., Hirota, Y., NishiHARA, E., OKUMURA, S., AND MORI, A. 1995 Near-IR Observation of Cometary Impacts to Jupiter: Brightness Variation of the Impact Plume of Fragment K. Publ. Astron. Soc. Japan 47, L21.

Weaver, H. A., A'Hearn, M. F., Arpigny, C., Boice, D. C., Feldman, P. D., Larson, S. M., Lamy, P., LevY, D. H., Marsden, B. G., Meech, K. J., Noll, K. S., et al. 1995 The Hubble Space Telescope (HST) Observing Campaign on Comet Shoemaker-Levy 9. Science 267, 1282.

West, R. A., Karkoschka, E., Friedson, A. J., Seymour, M., Baines, K. H., And Hammel, H. B. 1995 Impact Debris Particles in Jupiter's Stratosphere. Science 267, 1296.

Woodney, L. M., Meier, R., A'Hearn, M. F., Wellnitz, D., Smith, T., Verveer, A., AND MARTIN, R. 1995 Photometry of the Jovian Satellites During Comet D/ShoemakerLevy 9's Impact with Jupiter. DPS meeting abstract.

ZahnLe, K., AND MaCLow, M. M. 1994 The collision of Jupiter and Comet Shoemaker-Levy 9. Icarus 108, 1.

ZAHNLE, K., AND MACLow, M. M. 1995. A simple model for the light curve generated by a Shoemaker-Levy 9 impact. J. Geophys. Res. 100, 16885. 